\title{
Near wake of an impulsively started disk
}

\author{
H. Joharia) \\ Department of Mechanical Engineering, Worcester Polytechnic Institute, Worcester, Massachusetts 01609 \\ K. Stein \\ Department of Physics, Bethel College, St. Paul, Minnesota 55112
}

(Received 6 February 2001; accepted 1 July 2002; published 5 September 2002)

\begin{abstract}
The near wake of an impulsively started disk was studied computationally by a finite element code with a Smagorinsky turbulence model. The shear layer separating from the disk lip rolled up into a symmetric starting vortex ring at first. As time evolved, the vortex stretched in the downstream direction and flow instabilities caused the vortex ring to become wavy eventually leading to the breakup of the ring. The complete breakup and shedding of the starting vortex ring took a time of approximately $14 D / U$, where $D$ is the disk diameter and $U$ is the freestream velocity. The starting vortex ring circulation attained a plateau of $\approx 2.6 U \mathrm{D}$ at a time of about $4 \mathrm{D} / \mathrm{U}$, in good agreement with the experimental findings by Balligand (2000). The radial circulation profiles are Gaussian during the symmetric phase and collapse together at a time of $4 D / U$. Beyond this time, the vortex ring celerity is constant and vorticity extends to the symmetry axis. The base pressure coefficient becomes positive as the vortex ring moves away from the disk and remains positive until the ring is completely shed. The breakup of the starting vortex ring is concurrent with the appearance of an azimuthal pressure gradient and core flow. Following the breakup of the starting vortex ring, the flow became three-dimensional and settled into an open wake. The mean drag and base pressure coefficients were nearly constant after a time of approximately $30 \mathrm{D} / U$ and matched very well against experimental data. (C) 2002 American Institute of Physics. [DOI: 10.1063/1.1502267]
\end{abstract}

\section{INTRODUCTION}

The long-term objective of this effort is to get a better understanding of the unsteady flow in the near wake of a parachute canopy. The near wake is responsible for the aerodynamic forces and moments on the canopy, as well as the overall stability of the parachute system. It is well known that the wake of bluff bodies is three-dimensional and unsteady even when the body is planar or axisymmetric. Further complications arise when the interaction of the vortical flow with the flexible canopy fabric is considered. To simplify the flow and gain insight into the wake of an unsteadily moving bluff body, the impulsively started flow around an infinitesimally thin solid circular disk was pursued.

Although the steady flow about a disk has been examined by several investigators, ${ }^{1-3}$ the starting flow has received relatively little attention. The starting disk flow has been studied experimentally ${ }^{4-8}$ and numerically by discrete vortex methods. ${ }^{9,10}$ The experiments of Higuchi and co-workers ${ }^{4-7}$ were carried out in a tow tank at Reynolds numbers in the range of $10^{3}-10^{4}$. Flow visualization has revealed that initially a symmetric starting vortex ring is formed after which the ring experiences instability and becomes distorted. After the deformed vortex ring is shed, a steady-state wake structure is established. The axisymmetric discrete vortex method of Strickland ${ }^{9}$ was able to predict the drag history accurately during the starting phase of the flow,

a) Author to whom correspondence should be addressed. Telephone: (508) 831-5261; fax: (508) 831-5680; electronic mail: hjohari@wpi.edu and as long as the flow remained nearly axisymmetric. Shirayama and Kuwahara ${ }^{11}$ devised a discrete vortex method that is capable of nonaxisymmetric simulations. However, their computed drag for a disk under-predicted the experimental value by $25 \%$. Because of the prevalence of viscous effects near the disk, it appears that Navier-Stokes solvers are needed to accurately capture the complex viscous threedimensional (3-D) flow in the disk near wake. This is especially true after the starting vortex ring loses its symmetry.

A primary feature of the flow about the disk, and the analogous flat plate in two-dimensional flow, is that the separation is fixed at the disk edge. This feature creates a singularity around the edge in Navier-Stokes computations employing traditional grid-based schemes. Although vortex methods circumvent this difficulty, accurate solutions require the viscous effects to be accounted for during the wake development. To overcome the difficulties associated with the singularity at the disk edge and yet account for the viscous effects, a stabilized finite element formulation ${ }^{12}$ was utilized to solve the time-dependent, incompressible Navier-Stokes equations.

In the present study, the evolution of the near wake of an impulsively started disk from the formation of the starting vortex ring, to its breakup, and the eventual attainment of the steady state was simulated using a three-dimensional finite element code. The computed data not only provide the global quantities such as drag, but also allow the examination of the local flow features such as the base pressure coefficient and vorticity distribution within the starting vortex core. The circulation, energy, and impulse are computed during the wake 
development, and the formation of the starting vortex ring is addressed. The present results supplement and extend the experimental work of Higuchi and co-workers ${ }^{4-7}$ on the starting disk to a larger Reynolds number. Our 3-D data set further permits the examination of the flow during the transition from initial axisymmetry to three-dimensionality.

The computational work discussed here took advantage of the research code that is currently in use by the Airdrop Technology Team and was originally developed by Tezduyar. ${ }^{12}$ The numerical formulation is described in Sec. II. The results are discussed in Sec. III, and they are compared with those computed on a finer mesh in Sec. IV. The conclusions are presented in Sec. V. The computed data are compared with the experimental data available in the literature to the extent possible.

\section{NUMERICAL FORMULATION}

\section{A. Governing equations}

The incompressible Navier-Stokes equations were utilized in this study. Let $\Omega \subset R^{3}$ be the three-dimensional spatial domain. The spatial coordinates are denoted by $\mathbf{x}$ $=(x, y, z)$. The Navier-Stokes equations for incompressible flows are

$$
\begin{aligned}
& \rho\left(\frac{\partial \mathbf{u}}{\partial t}+\mathbf{u} \cdot \boldsymbol{\nabla} \mathbf{u}+\mathbf{f}\right)-\boldsymbol{\nabla} \cdot \boldsymbol{\sigma}=0, \quad \text { in } \Omega, \\
& \boldsymbol{\nabla} \cdot \mathbf{u}=0, \quad \text { in } \Omega,
\end{aligned}
$$

where $\rho, \mathbf{u}, \mathbf{f}$, and $\boldsymbol{\sigma}$ are the density, velocity, body force, and stress tensor, respectively. The stress tensor for a Newtonian fluid with dynamic viscosity $\mu$ and the strain rate tensor $\boldsymbol{\varepsilon}(\mathbf{u})$ is defined as follows:

$$
\boldsymbol{\sigma}(\mathbf{u}, p)=-p \mathbf{I}+2 \mu \boldsymbol{\varepsilon}(\mathbf{u}),
$$

where $p$ is pressure and $\mathbf{I}$ is the identity tensor. The various terms in the Navier-Stokes equations were nondimensionalized by the fluid density, disk diameter, and freestream velocity.

For the flow under consideration, $\mu$ was modified locally using a Smagorinsky turbulence model ${ }^{13}$ as follows:

$$
\mu_{\text {modified }}=\mu+\rho\left(C h_{e}\right)^{2} \sqrt{2 \varepsilon: \varepsilon} .
$$

Here, $C$ is a constant equal to 0.15 and $h_{e}$ represents the element length scale. The variation of $h_{e}$ depends on the local mesh refinement. The Smagorinsky model was chosen because of its efficiency at high Reynolds numbers. The initial condition for the velocity was specified as $\mathbf{u}(\mathbf{x}, 0)=\mathbf{u}_{0}$ in $\Omega$, where $\mathbf{u}_{0}$ was divergence-free.

The solutions presented in this paper were obtained using a stabilized semi-discrete formulation for the NavierStokes equations. The details of this formulation and its implementation can be found in Refs. 12 and 14. This formulation is particularly well suited for time-dependent flows and has been applied to a variety of 3-D flows. The code has been validated in several flow fields, particularly for the wake of a circular cylinder. ${ }^{15,16}$ The formulation, which is consistent with the Galerkin formulation, was chosen because it reduces numerical oscillations without introducing

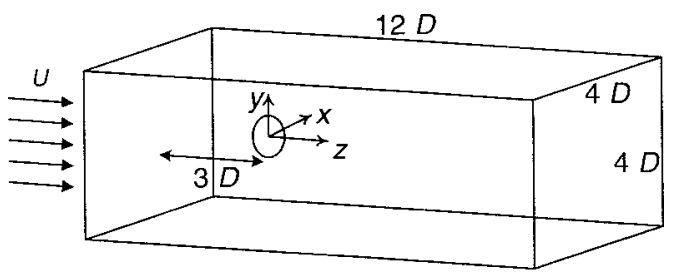

FIG. 1. Schematic of the computational domain and the coordinate system used.

excessive numerical dissipation in incompressible flows at high Reynolds numbers. Essentially the formulation is stabilized against numerical instabilities that arise in advectiondominated flows. In the semi-discrete formulation the timeintegration is achieved with central differencing. For the solutions presented here, we utilized an unstructured mesh of linear tetrahedral elements for spatial discretization.

\section{B. Numerical model}

The computational domain along with the disk of diameter $D$ is shown schematically in Fig. 1. The domain consisted of a rectangular volume having a square cross-section of $4 D$ on each side and $12 D$ long. The infinitesimally thin disk was located $3 D$ from the inflow. At the inflow, a uniform velocity was prescribed. At the outflow, a traction-free boundary condition $(\hat{\mathbf{n}} \cdot \boldsymbol{\sigma}=0$, where $\hat{\mathbf{n}}$ is the unit normal vector) was imposed. Free-slip boundary conditions $(\hat{\mathbf{n}} \cdot \mathbf{u}=0)$ were prescribed on the four sides. The disk surface was treated as a no-slip boundary. The origin of the Cartesian coordinate system was placed at the disk center with the $z$-axis, which was the disk symmetry axis, in the freestream direction.

A tetrahedral volume mesh was generated using unstructured mesh generation software based on the strategies described in Johnson and Tezduyar. ${ }^{17}$ The mesh consisted of 708,594 elements with 114,500 nodes. Mesh refinement was utilized on a rectangular volume of $1.5 D$ square crosssection extending from $1.5 D$ upstream to $5 D$ downstream of the disk. The mesh edge lengths in this region ranged from about 0.05 to $0.08 D$. The choice of mesh density was based on the results of a resolution study in which increasing this mesh density by a factor of two did not produce any significant changes to the flow evolution or the initial and steadystate drag values. The adequacy of the mesh density is also demonstrated by the close agreement between the computed drag and base pressure coefficients with the experimental data in the literature. Therefore, the chosen mesh density was deemed sufficient to resolve the primary flow features in the near wake during the startup and steady-state periods. The data from the mesh refinement study are discussed in Sec. IV.

In the computations, the flow about the disk was impulsively started by switching the inflow velocity $U$ from zero to one in the first time step. The rapid change in the inflow velocity created oscillations which subsided in the first 10 time steps. The Reynolds number, $\operatorname{Re}=U D / \nu$, was set at 2 $\times 10^{5}$ by fixing the value of viscosity $\nu$. The parameters were chosen so that the flow development at high Re could be examined. In an experiment at this Re, the boundary layer on 
the disk upstream side could be transitional or turbulent, especially if freestream turbulence is present. However, we do not expect that the large-scale flow features to be affected significantly since our computed drag and base pressure coefficients are in good agreement with experimental data at an even higher Reynolds number.

A temporal resolution study was performed in which the time steps ranged from $0.0025 D / U$ to $0.025 D / U$. Only very minor quantitative differences were observed when the time step was changed. A time step of $0.01 D / U$ was chosen in order to keep the total computational time reasonable. The computations were run for 6000 time steps resulting in the flow evolution in the first $60 D / U$ after the flow initiation. The code was implemented using a message-passing paradigm, and the computations were carried out using 32 processors on a CRAY T3E-1200.

\section{RESULTS}

The evolution of the impulsively started flow around the disk can be divided into three phases based on the drag and flow characteristics. The first phase, which started from the onset of simulation, was characterized by the formation, growth, and breakup of a starting vortex ring in the near wake region. The second phase covered the transition from the starting flow to the steady-state phase. In the third phase, the flow settled in its steady state with a quasi-periodic vortex shedding. In this phase, the drag and base pressure were nearly constant, and the pressure distribution on the backside of the disk was approximately uniform. Velocity vectors in a cross-sectional plane during the startup and the steady-state phases of the flow evolution are presented in Fig. 2. The disk had traveled $1 D$ and $52 D$ in the flow fields shown in Figs. 2(a) and 2(b), respectively. Although the two flow fields are quite similar on the upstream side of the disk, the near wake regions are quite different. The presence of the starting vortex ring created reverse flow within a recirculation bubble that terminated at a stagnation point in the startup velocity field. On the other hand, the separated flow has caused a large open wake with relatively small velocities in the steady-state phase. Various aspects of the startup and steadystate phases are discussed in detail in the following sections.

\section{A. Startup phase}

The separated flow around the perimeter of the disk rolled up into a symmetric vortex ring, which grew by the accumulation of vorticity shed from the disk lip. As time evolved, the vortex ring moved away from the disk and its circulation increased. Eventually, the vortex ring became unstable, broke apart, and its remnants were shed. The pressure distribution on the back of the disk and the drag were strongly affected by the strength and position of the vortex ring in the startup phase.

To visualize the external structure of the starting vortex ring, we utilize iso-pressure surfaces. The advantage of displaying an iso-pressure surface instead of components of the vorticity vector is masking of the separated shear layer. The evolution of the starting vortex ring is shown in a sequence of images in Fig. 3. The pressure coefficient, $C_{p}$, of the

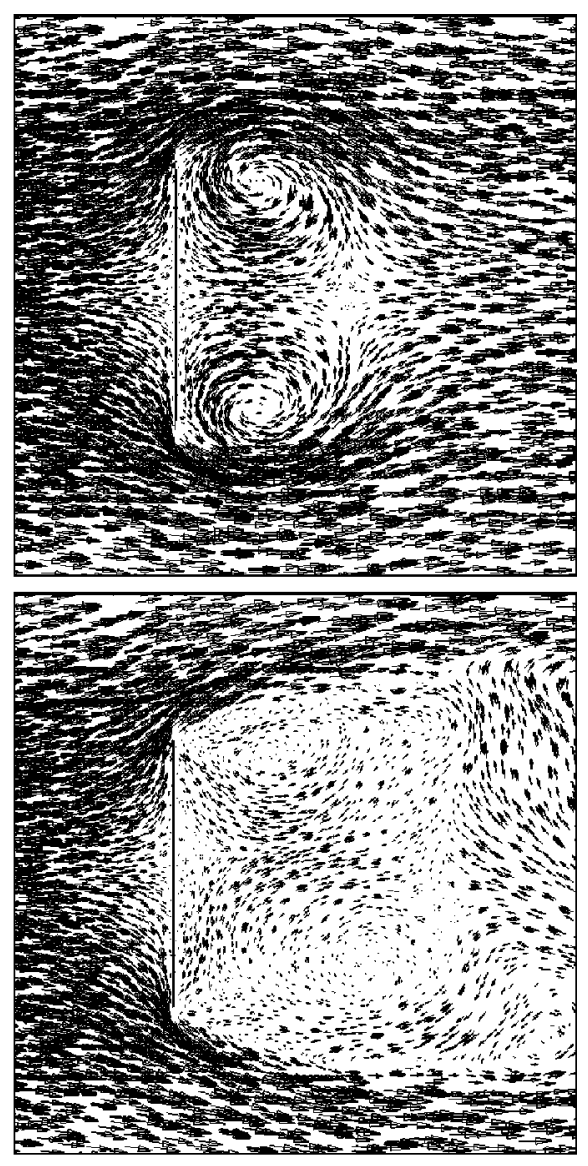

FIG. 2. The velocity vectors in a cross-sectional plane during the startup (a), and steady-state (b) phases of the flow.

rendered surface was chosen to be -1.72 , corresponding to $90 \%$ of the minimum $C_{p}$ in the vortex core at time $T$ $\equiv t U / D=1.0$. The vortex ring remained quite symmetric up to a time of $T \approx 7$, after which the ring experienced the Widnall ${ }^{18}$ instability and waves appeared on the ring. Only a few waves are present on the ring due to the relatively thick vortex core. The increasing amplitude of the waves severely distorted the ring, and one side of the vortex ring became closer to the disk. The appearance and growth of the waves in free vortex rings is followed by a rapid breakdown. ${ }^{19}$ Here, we also see that the upper part of the vortex ring in Fig. 3 was pinched-off at a time of $T \approx 12$, and the ring broke up by $T \approx 13$. Complex vortex interactions ensued and the remnants of the starting vortex ring were completely shed by $T=15$. After the onset of asymmetry, azimuthal pressure differences along the core appeared due to the waves on the ring. This issue will be discussed further in Sec. IIIE as it relates to the breakup of the ring.

The evolution of the disk drag coefficient $C_{D}$ and base pressure coefficient $C_{p b}$ (at the disk center) during the startup phase are plotted in Fig. 4. Drag, calculated from the integration of pressure difference across the disk, was very large initially due to the impulsive start. The pressure distribution on the upstream side of the disk remained nearly constant throughout. On the other hand, pressure on the downstream side of the disk was strongly affected by the starting vortex. As the ring moved away from the disk, the base 


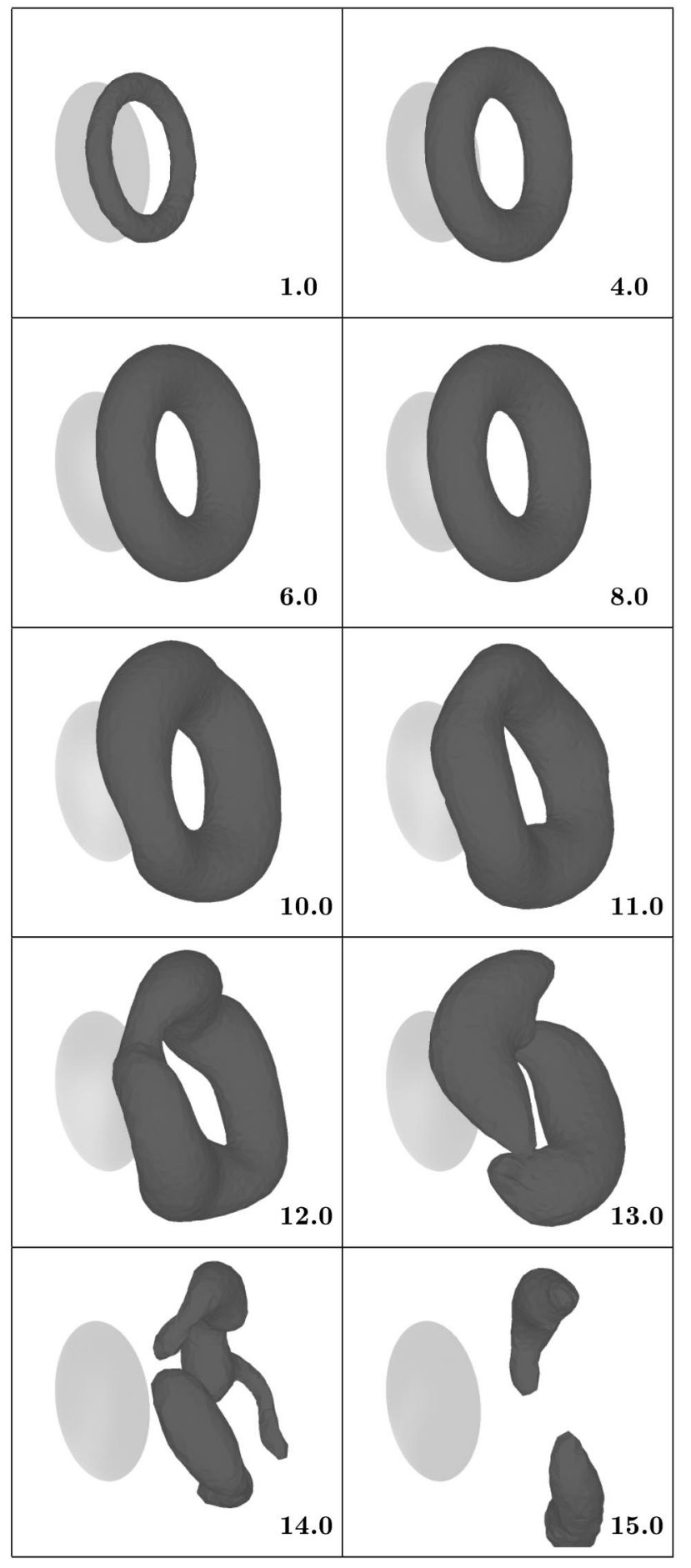

FIG. 3. Evolution of the starting vortex ring as visualized by iso-pressure surfaces. The numbers indicate the nondimensional time $T$.

pressure increased leading to a reduction in drag. Drag coefficient reached a minimum of 0.65 as the base pressure coefficient increased to a positive value of 0.35 at $T \approx 9.5$. The highest surface $C_{p}$ was generally at the disk center until appreciable asymmetry had appeared. When the vortex ring broke apart, the base pressure decreased and the drag increased.

Drag data from the experiments of Higuchi et al. ${ }^{10}$ are

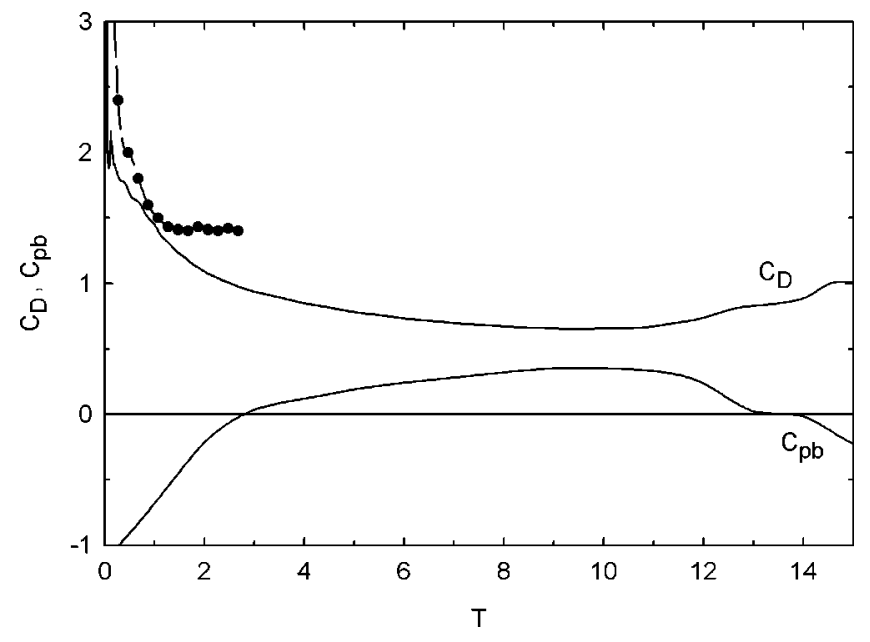

FIG. 4. Drag and base pressure coefficient during the startup phase. The symbols represent experimental data from Ref. 10.

also included in the plot of Fig. 4. The experimental data which had a linear ramp-up time of $T=0.33$ reached its maximum $C_{D}$ at a time of $T \approx 0.3$. To compare the impulsively started computed flow against the experiments with the finite ramp-up time, the experimental data were shifted in time so that the time corresponding to the initial drag peak coincided with the first time step. The observed decrease in the drag coefficient is quite consistent with the experimental data. Unfortunately, the experiments were not continued for a longer time.

In addition, a comparison of our results with those of Chua et al. ${ }^{20}$ where the starting flow about a flat plate was studied, suggests general agreement in drag behavior. Both the experiments and the discrete vortex method simulations of Chua et al..$^{20}$ showed a minimum in the drag at a nondimensional time of 8 . The starting vortex pair behind the plate broke up at a time of 10. These times are in accord with the analogous times in our computations of the starting disk. The more recent 2-D numerical study of the impulsively started flat plate by Koumoutsakos and Shiels ${ }^{21}$ has also showed a rapid decrease in the drag coefficient. However, no minimum in the drag was observed because of the persistence of the 2-D starting vortex.

A consequence of the presence of the starting vortex ring is the formation of a recirculation bubble with a stagnation point away from the disk surface. The stagnation point is clearly discernible in the axisymmetric flow field of Fig. 2(a). The bubble grew as the starting vortex ring expanded. The stagnation point remained on the symmetry axis until a time of $T \approx 10$, after which it moved off the $z$-axis and disappeared at $T \approx 13$, corresponding with the breakup of the ring. The evolution of the bubble length, $s$, as quantified by the stagnation point, is shown in Fig. 5. The bubble length increased to $1.5 \mathrm{D}$ while the flow was axisymmetric. The linear increase of the bubble length in the time span of 4 $\leqslant T \leqslant 12$ indicates that the stagnation point was moving with a constant speed in this period. The experimental data of Balligand $^{5,6}$ is also plotted for comparison. Initially there is good agreement; however, the computed bubble length increases more rapidly than in the experiments. At the last 


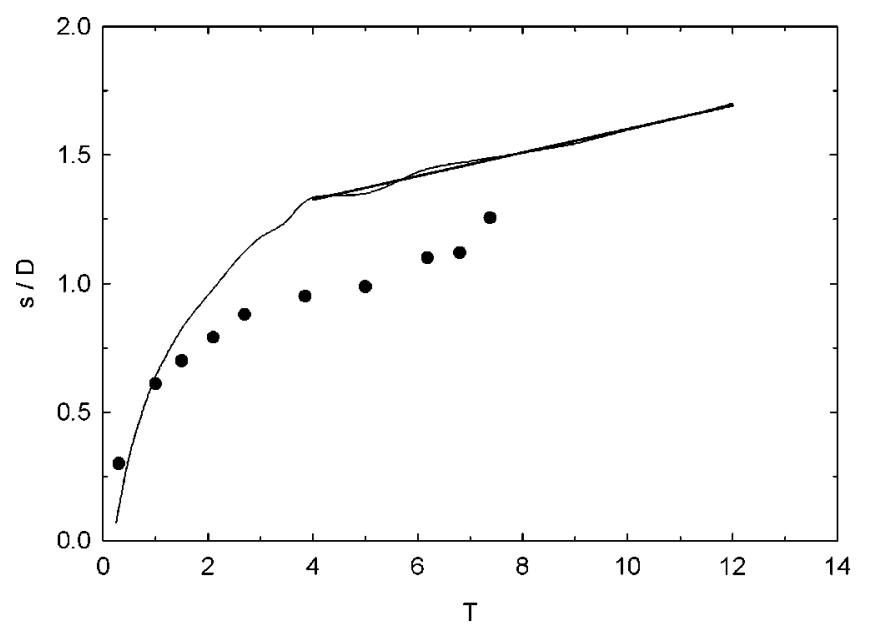

FIG. 5. Recirculation bubble length. Symbols are experimental data from Ref. 6 . The bold straight line indicates the linear growth of the wake bubble.

reported data point, the measured bubble length was approximately $15 \%$ smaller than that in our simulation. The lower Reynolds number of the experiments $\left(\approx 10^{4}\right)$ is suspected to be the main reason for the observed differences.

The minimum pressure in the stating vortex core is plotted in Fig. 6 at four azimuthal angles around the vortex. The pressure coefficient in the vortex core decreased continually from -1.9 at $T=1$ to -2.3 , beyond which large amplitude oscillations appeared due to the onset of instability waves. The minimum $C_{p}$ in the vortex core could not be followed beyond the time of $T=14$. The initial decrease in the minimum $C_{p}$ indicates the strengthening of the vortex ring.

The minimum $C_{p}$ location was identified as the core of the starting vortex. The normalized axial, $z_{\text {core }} / D$, and radial, $r_{\text {core }} / D$, coordinates of the vortex core are plotted in Fig. 7 at four azimuthal angles around the ring. The symmetry of the vortex ring is evident in the close proximity of the data up to $T \approx 7$. The onset of instability waves and ring breakup beyond $T=13$ is indicated by the appreciable upstream and inward movement of the ring segment near $90^{\circ}$. For comparison purposes, the experimental data of Balligand and Higuchi $^{4}$ at $\mathrm{Re} \approx 10^{4}$ are included in the plot as well. The

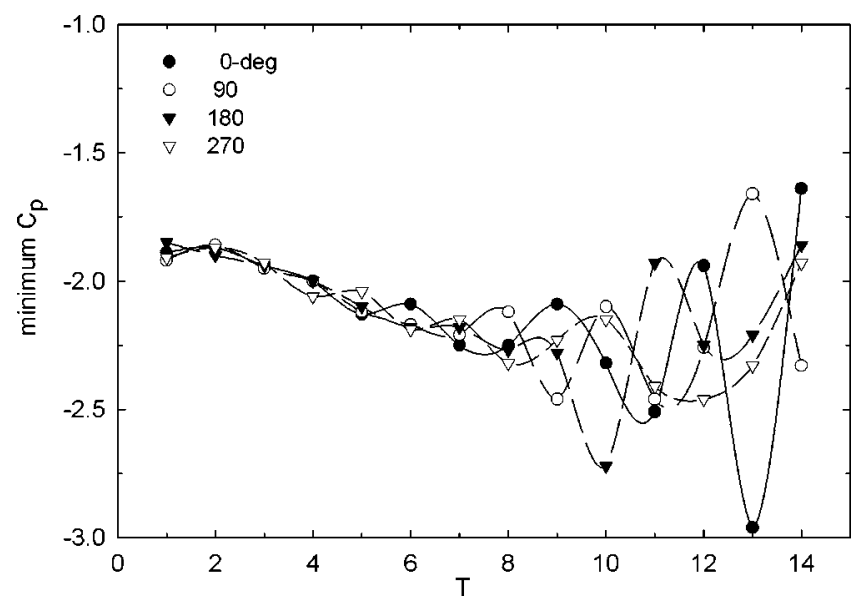

FIG. 6. Minimum pressure coefficient in the starting vortex core at four azimuthal angles.
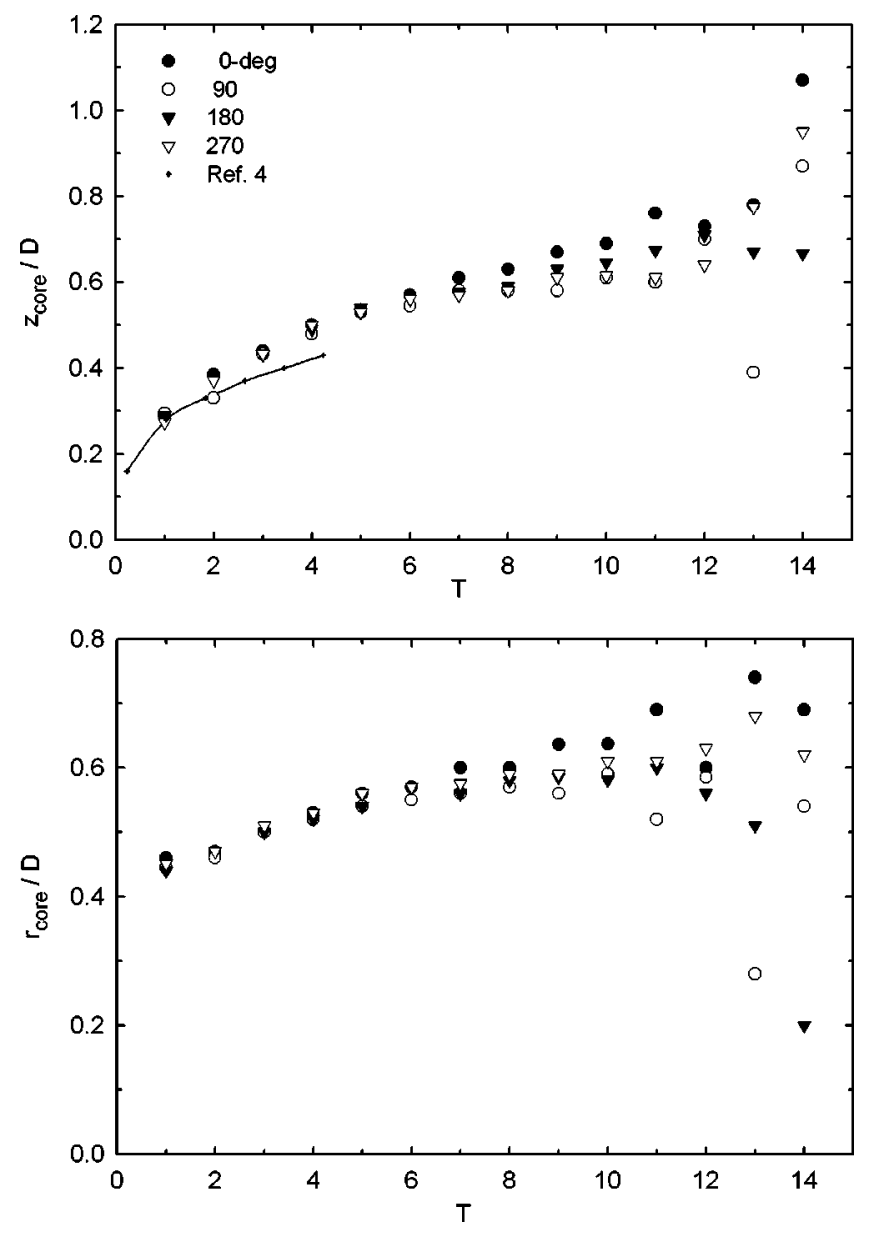

FIG. 7. Axial and radial position of the starting vortex core based on the minimum $C_{p}$ location. The curve represents experimental data from Ref. 4 .

experimental run had a linear ramp-up time of $T=0.56$ and the vortex core locations were extracted from the center of fluorescent dye (illuminated by a laser sheet) images. The experimental data were shifted in time to account for the ramp-up time. There appears to be good agreement in the early stages of vortex ring development. As time evolved, the computed vortex ring moved farther than the experimental one. At $T=4$, the computed vortex was about $0.07 D$ farther downstream of the experimental vortex. It should be noted that a more appropriate comparison would be between the streakline (emanating from the disk lip) patterns from the computed data and the experimental data of Ref. 4. The radial position of the starting vortex center in the experiments ranged from 0.48 to $0.58 D$ up to a time of $T=6$, which is in close agreement with the present computations.

A different perspective of the starting flow is provided by the vorticity field. The azimuthal component of vorticity field is shown in Fig. 8 in two perpendicular planes. The images on the left side correspond to the vorticity in the $y-z$ plane whereas the images on the right side are from the $x-z$ plane. The $x-z$ and $y-z$ planes relate to horizontal and vertical cuts in the iso-pressure surfaces of Fig. 3. The darkest shades indicate the highest absolute values of azimuthal vorticity. The vorticity in the disk boundary layer is also visible in the images of Fig. 8. As time evolved, the concentrated 

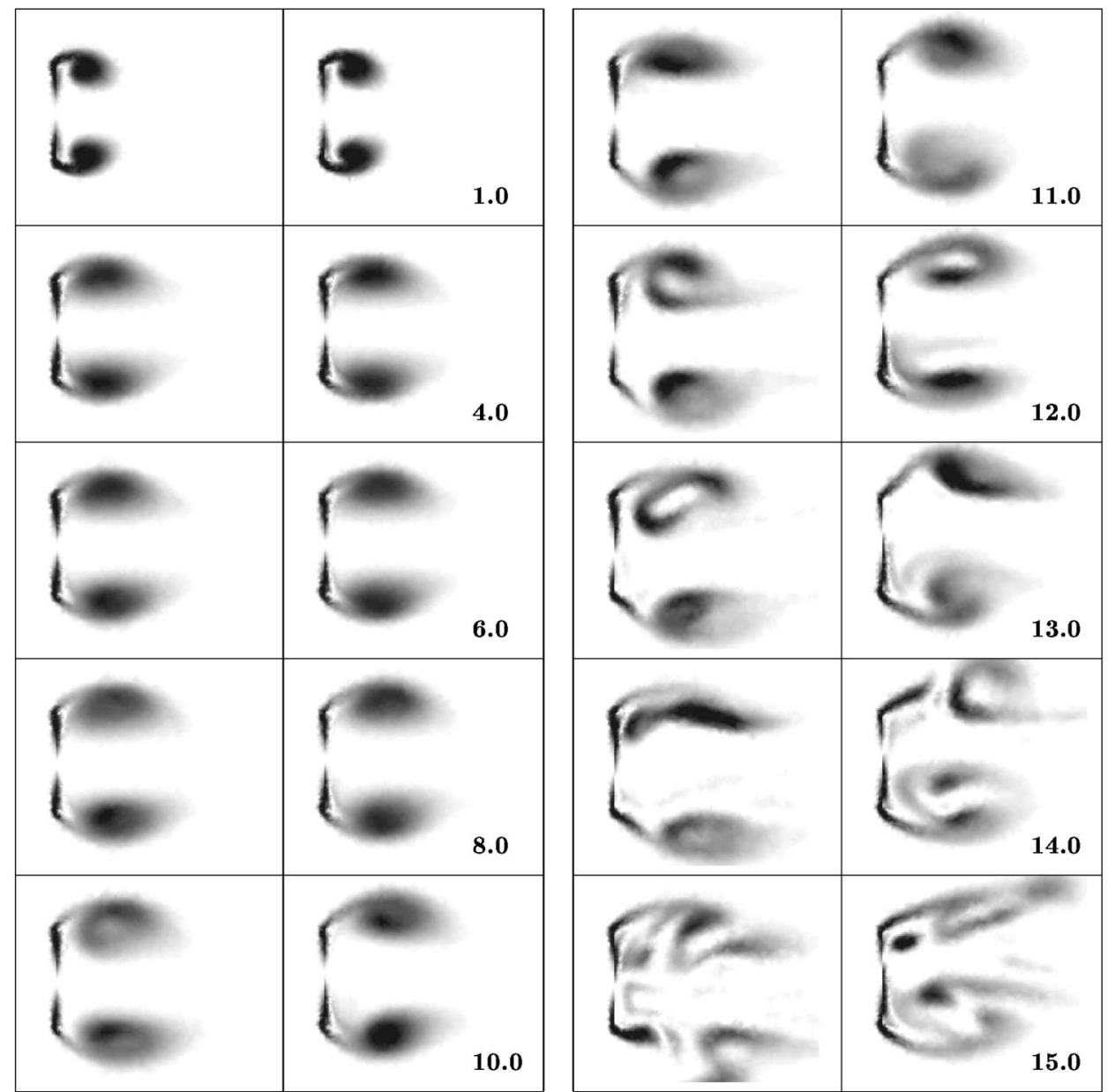

FIG. 8. Evolution of the azimuthal vorticity field in two perpendicular planes. The numbers indicate the nondimensional time $T$. vorticity field of the vortex ring diffused and the peak vorticity decreased. The stretching of vorticity in the axial direction is especially notable. It is evident that the vorticity field was axisymmetric up to a time of $T=6$, consistent with the pressure surface rendering. Asymmetry of the vorticity field became noticeable by $T \approx 7$. The upper portion of the vortex ring in the $y-z$ plane became larger and more diffuse than the lower portion at $T=10$. The vortex cores became elongated in the $y-z$ plane at the time of $T=11$. Meanwhile the lower vortex core in the $x-z$ plane had enlarged and moved closer to the disk when compared to the upper core in the same plane. The vorticity field had lost its ring structure by $T \approx 13$. The remnants of the starting vortex were shed at $T \approx 15$ and smaller vortices formed near the disk. After the starting vortex was shed, streamwise vorticity filaments became prominent in the near wake. The exact time of the onset of asymmetry and the topology of the ring breakup depends somewhat on the mesh refinement; however, the overall computed features and trends are shown to be mesh independent by the refined mesh data presented later.

Similar features have been observed in the flow visualization experiments of Balligand and Higuchi with a starting disk (see Figs. 57, 58, 60, and 63 in Ref. 4). The asymmetry and breakup of the vortex ring in the experiments generally started a little sooner than in the present computations although in one run (see Fig. 63 in the Ref. 4) the times were comparable. The tilting and breakup of the vortex ring oc- curred in random orientations in the experiments.

The maximum azimuthal vorticity in the vortex core is plotted in Fig. 9 at four azimuthal angles around the vortex. The vorticity in the shear layer rolled up into a vortex core shortly after the onset of motion. The peak azimuthal vorticity decreased continuously due to the diffusion of vorticity as the axisymmetric vortex expanded. The close agreement of the vorticity values prior to the onset of instability is note-

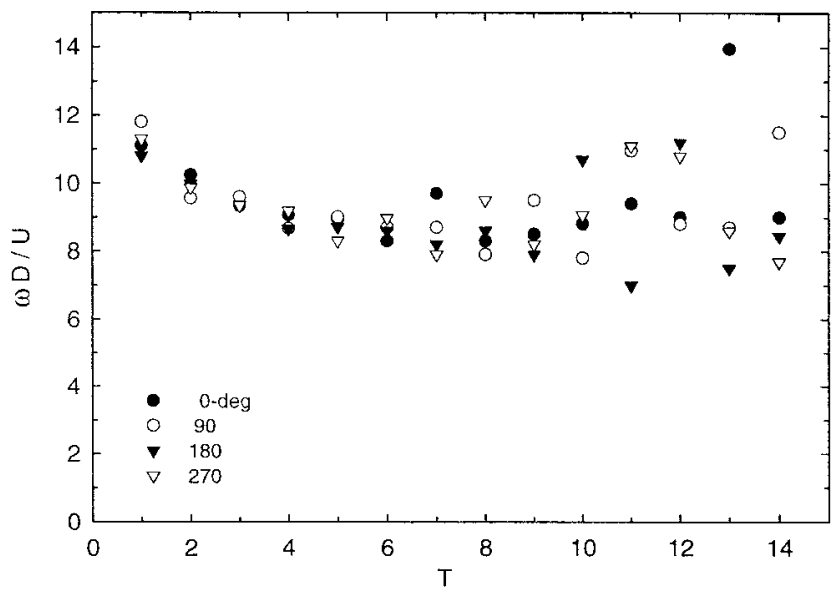

FIG. 9. Maximum azimuthal vorticity in the starting vortex core at four azimuthal angles. 

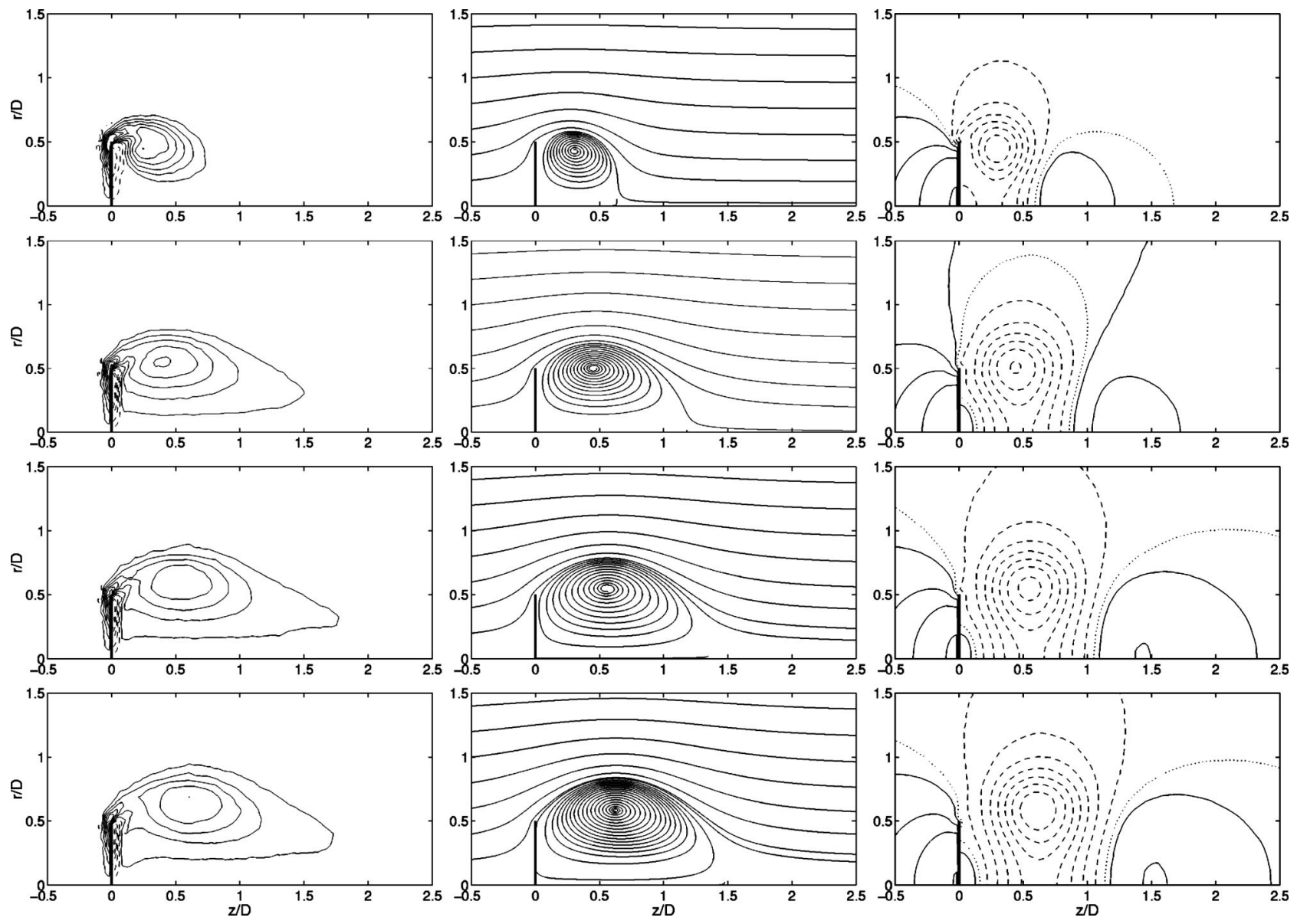

FIG. 10. Vorticity contours (left), streamlines (middle), and pressure contours (right) at $T=1,3,5$, and 7 (top to bottom). Positive and negative contours are indicated by solid and dashed curves, respectively. Minimum $|\omega| D / U$ contour is 1 with an increment of $2 . C_{p}$ contours start at 1 and decrease by 0.3 . The zero $C_{p}$ contour is the dotted curve.

worthy. The divergence of the peak vorticity values around the ring starts at $T \approx 7$, in excellent agreement with the data in the minimum $C_{p}$ plot of Fig. 6 . The axial core location based on the maximum vorticity was quite similar to that obtained from the minimum pressure data shown in Fig. 7. However, the ring diameters based on the peak vorticity were larger than those obtained from the minimum pressure.

\section{B. Axisymmetric period}

The details of the initial axisymmetric flow are examined in Fig. 10 through the development of the vorticity and pressure contours as well as the instantaneous streamlines. Time instants of $T=1,3,5$, and 7 are considered. The separated flow at the lip rapidly rolled up into a vortex ring at first. A pressure gradient on the back of the disk, induced by the vortex ring, created a region of secondary vorticity. The dashed vorticity contours indicate the opposite-signed vorticity. As the vortex strengthens, so does the secondary vorticity on the disk. The interaction of this secondary vorticity with the primary shear layer vorticity appears to annihilate vorticity near the disk lip. Furthermore, there seems to be interaction between the two opposite-signed vorticity along the majority of the disk backside as revealed by the close proximity of the positive and negative contours there.

As the starting vortex evolved, its axial extent became much larger than its radial and the vorticity contours took on an elliptical cross section. The stretching of the vortex results in the elongation of the wake bubble discussed earlier. The similarity of the vorticity contours at $T=5$ and 7 is noteworthy. The relative stationarity of the higher vorticity contours at these times suggest that the starting vortex is fully formed by $T=5$. This is corroborated by the data and discussion in a subsequent section.

Previous work ${ }^{6-8}$ has reported the formation of discrete vortices in the shear layer forming the starting vortex. These smaller discrete vortices have been attributed to the KelvinHelmholtz instability of the shear layer. Koumoutsakos and Shiels ${ }^{21}$ in their high resolution study of the starting flat plate also observed such discrete vortices, but only for the uniformly accelerated case. The impulsively started flat plate in Ref. 21 did not produce any discrete vortices in the shear layer. Pullin and Perry ${ }^{22}$ argue that the instability is triggered by external disturbances in the experiments. The lack of discrete vortices in our simulation is perhaps due to the inadequate spatial resolution and/or minimal numerical disturbances in the initial period.

The instantaneous streamlines at the above mentioned time instants are also presented in Fig. 10. The development of the wake bubble is characterized by the rapid axial elongation and the slower radial expansion. The positive and 


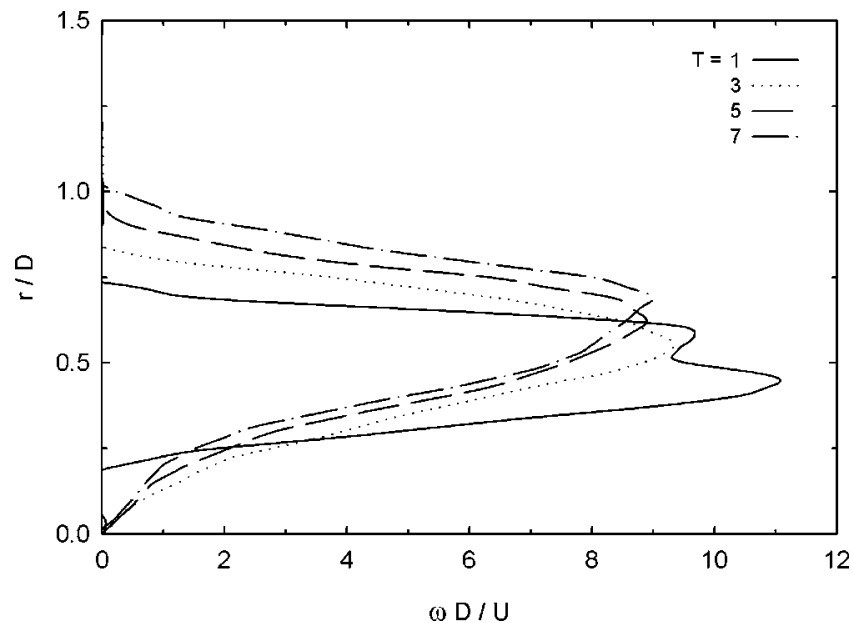

FIG. 11. Radial profiles of vorticity through the peak vorticity position at $T=1$ (solid), 3 (dotted), 5 (dashed), and 7 (dash-dotted).

negative pressure contours in Fig. 10 are denoted by solid and dashed curves, respectively. At $T=1, C_{p}$ on the entire backside of the disk is negative. As time evolved and the vortex core moved away from the disk, $C_{p}$ became positive over the central portion of the disk backside (see $T=3$ ). The large negative $C_{p}$ region associated with the starting vortex is followed by a compression zone with positive $C_{p}$ values. Pressure recovers to the freestream value after this compression zone. Note that analogous to the vorticity contours, the pressure contours are similar at $T=5$ and 7 .

The radial profiles of vorticity at four instants during the axisymmetric period is shown in Fig. 11. The profiles are through the peak vorticity location. At $T=1$, there were two peaks in the profiles due to the presence of vorticity not yet rolled up into the core. The profiles became Gaussian-like as time increased. At the same time, both the vortex radius and core thickness increased. For $T>3$, the vorticity profiles extended all the way to the symmetry axis revealing the axistouching nature of the starting vortex. For $T>5$, the ring is quite thick since the entire vorticity containing core diameter $\sim 1 D$ is comparable to the ring diameter of $1.2-1.4 D$.

Because of the initial symmetry of the vortex ring, an azimuthally-averaged vortex core trajectory was computed from both the vorticity and pressure fields. The average core trajectory is plotted in Fig. 12 from the initial roll-up to the time of $T=7$. The data from the pressure and vorticity fields correspond to the same time instants during the wake development. After $T=1$, the vortex core positions found from the minimum pressure are inward of those obtained from the peak vorticity. It is also evident that the vortex core contracted at first $(T<1)$, before increasing in the radial direction. The vortex core moved about $0.6 \mathrm{D}$ away from the disk before the ring instability appeared. The mean vortex trajectory (as determined by either maximum vorticity or minimum pressure) is linear after the ring began to expand. The growth rate $\left(d r_{\text {core }} / d z_{\text {core }}\right)$ found from the maximum vorticity was greater than that for the minimum pressure.

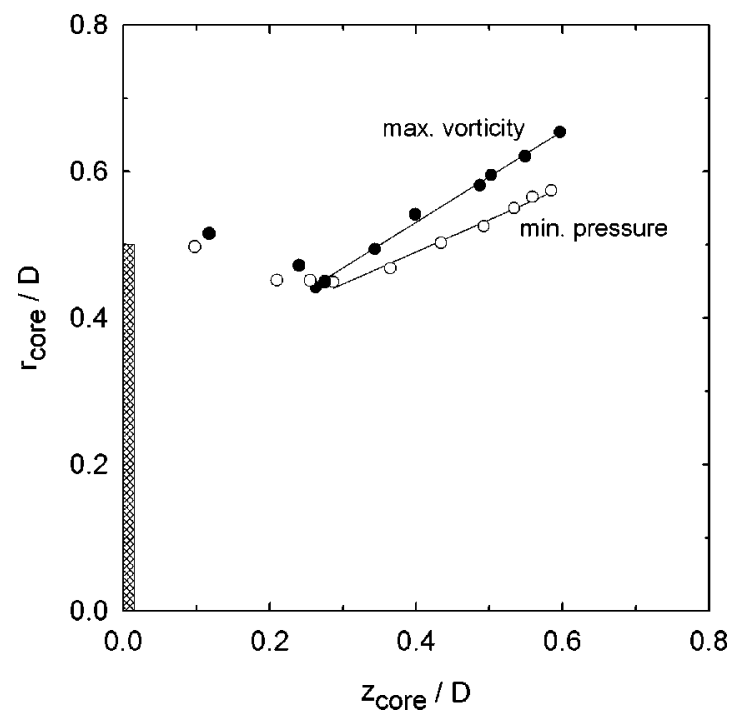

FIG. 12. Mean vortex core trajectory during the axisymmetric period. The filled symbols are based on the maximum vorticity and the open symbols on the minimum $C_{p}$.

\section{Integral measures}

The circulation, energy, and impulse of the developing wake were computed during the startup phase of the flow. The circulation was calculated from the line integral of velocity along iso-vorticity contours. The circulation development for three different iso-vorticity contours of 2.0, 1.0, and $0.2 U / D$ is shown in Fig. 13 while the wake was axisymmetric. The lowest contour is approximately equal to $2 \%$ of the maximum vorticity in the starting vortex core. A further reduction of the contour level to $1 \%$ of peak vorticity resulted in nearly the same values of circulation. As expected, the total wake circulation increases with time, the increase being nonlinear for all three contours. Also, circulation at any instant decreases as the iso-vorticity contour value increases. Beyond $T \sim 4$, the difference between the wake circulation at 1.0 and $2.0 U / D$ remains nearly constant, whereas the differ-

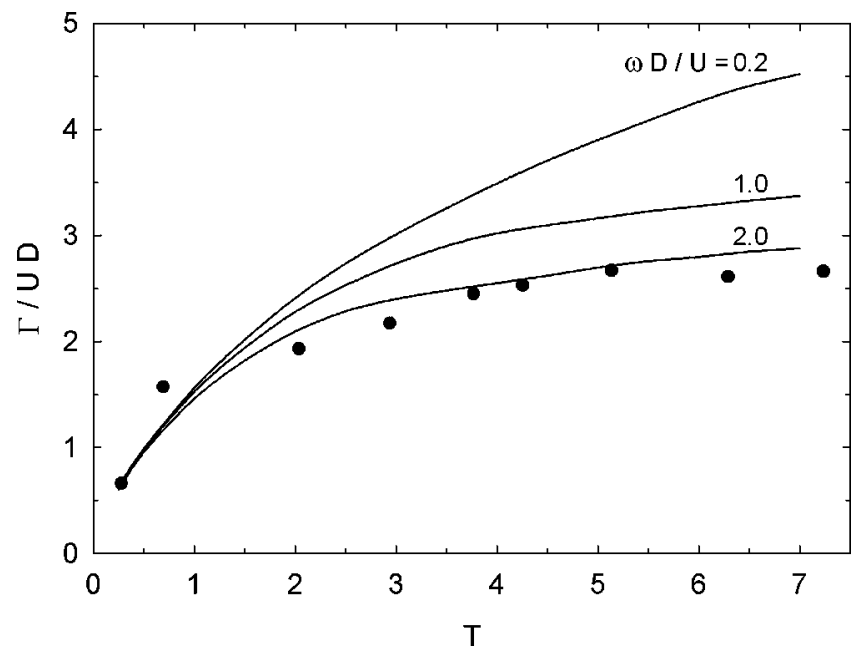

FIG. 13. The total circulation in the wake for three different integration contours. Symbols are experimental data from Ref. 6 computed on a rectangular contour. 
ence between the circulation for the $0.2 U / D$ and the other two contours keeps increasing. This is due to the "leakage" of vorticity from the higher vorticity levels to the lower ones. Prior to $T=1$, the 3 contours in Fig. 13 provide nearly equal circulation values because all the vorticity is confined to the higher contours. Diffusion has not had a chance to spread the vorticity yet. As time evolves, vorticity spreads to contours with lower vorticity values resulting in an expanded shear layer and vortex ring.

The experimental data in Refs. 5-7 corresponding to their largest Reynolds number of $1.2 \times 10^{4}$ with a ramp-up time of 0.33 are also included in Fig. 13. There is good agreement between the measured and computed data at the $\omega=2.0 U / D$ contour level. However, the experimental data indicate smaller circulation values than at the two lower isovorticity contours. The difference in circulation values stems from the different integration contours employed. A rectangular contour, containing the secondary vorticity of opposite sign in the back of the disk, was used in the experimental study in contrast to the iso-vorticity contours used here. The inclusion of opposite-signed vorticity would reduce the total circulation of the wake. Choosing a contour similar to that used in Ref. 6 would reduce our circulation values at the lower vorticity contours by about $20 \%$, and bring them closer to the experimentally measured values.

The rate of deposition of circulation into the wake can be quantified by the flux of vorticity at the disk edge. If we assume that the azimuthal vorticity can be approximated by $-(\partial u / \partial y)$, i.e. the "slug-flow" assumption, ${ }^{23}$ then the flux of vorticity becomes

$$
\frac{d \Gamma}{d t} \approx \frac{1}{2} u^{2} .
$$

For an impulsively started disk, this results in a total wake circulation that increases linearly with time at a rate of $U^{2} / 2$. The normalized wake circulation $\Gamma / U D$ should then be $\approx T / 2$. We can clearly see from the data in Fig. 13 that this estimate is not appropriate for the disk. The wake circulation is underestimated by a factor of 2 initially, and by about $30 \%$ by the time of onset of wake asymmetry. A similar failure of the slug-flow assumption for the disk wake was also noted in Ref. 6. The reasons for the shortcoming of the above estimate, especially initially, are discussed by $\operatorname{Didden}^{24}$ in his work on the formation of free vortex rings. The reduction in the difference between the estimated value and the circulation computed with the lowest vorticity contour can be attributed to the interaction of opposite sign vorticity on the back of the disk with the shear layer vorticity.

The wake energy and the axial component of impulse were calculated from the following expressions:

$$
\begin{aligned}
& E=\frac{1}{2} \rho \int \mathbf{u}^{2} d V, \\
& I=\frac{1}{2} \rho \hat{\mathbf{k}} \cdot \int \mathbf{x} \times \boldsymbol{\omega} d V .
\end{aligned}
$$

The integration was carried out over all the elements in the wake where the vorticity level was greater than $2 \%$ of the peak vorticity in the vortex core. Changing the limiting vor-

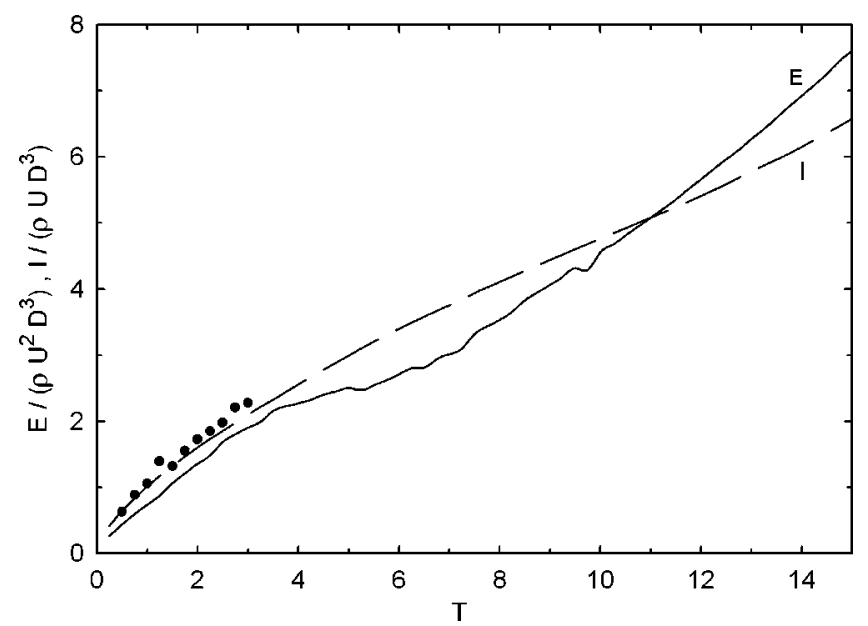

FIG. 14. Energy (solid curve) and impulse (dashed curve) development in the startup phase. Experimental data from Ref. 6 are represented by symbols.

ticity level did not have a significant effect on the results. For example, increasing or decreasing the limiting vorticity level by a factor of 2 changed the impulse by less than $2 \%$.

The development of normalized energy, $E / \rho U^{2} D^{3}$, and impulse, $I / \rho U D^{3}$, is shown in Fig. 14. Both energy and impulse increase with time, as expected. Although the wake impulse increases monotonically as $T^{0.7}$ in the entire startup phase, the rate of energy increase gets reduced at $T \sim 5$ before increasing again. The wake energy increases almost linearly for $T>9$. The change in the energy behavior coincides with the appearance of the asymmetry in the wake. The experimental impulse of Ref. 6 is also included in this figure. There is good correspondence between the computed results and the experimental data.

If the slug-flow concept is applied to the impulsively started disk problem, both the normalized energy and impulse should increase linearly with time, ${ }^{25}$

$$
\begin{aligned}
& \frac{E}{\rho U^{2} D^{3}} \approx \frac{\pi}{8} T, \\
& \frac{I}{\rho U D^{3}} \approx \frac{\pi}{4} T .
\end{aligned}
$$

Again, we see that the slug-flow is clearly inadequate for describing the wake energy and impulse development during the startup phase.

\section{Starting vortex formation}

Several recent studies ${ }^{25-27}$ have investigated the formation of vortex rings issued from piston/cylinder generators. It has been found that the vortex ring attains its eventual circulation by the time a stroke ratio of approximately 4 is reached. For longer stroke ratios, the excess circulation forms a trailing jet. We can also ask whether the circulation from the first $4 D$ travel of the disk is equal to the ultimate circulation of the starting vortex ring. To assess the circulation associated with the vortex ring alone, circular contours centered on the peak vorticity were utilized. Assuming that the vortex ring has a circular cross-section, at least initially 


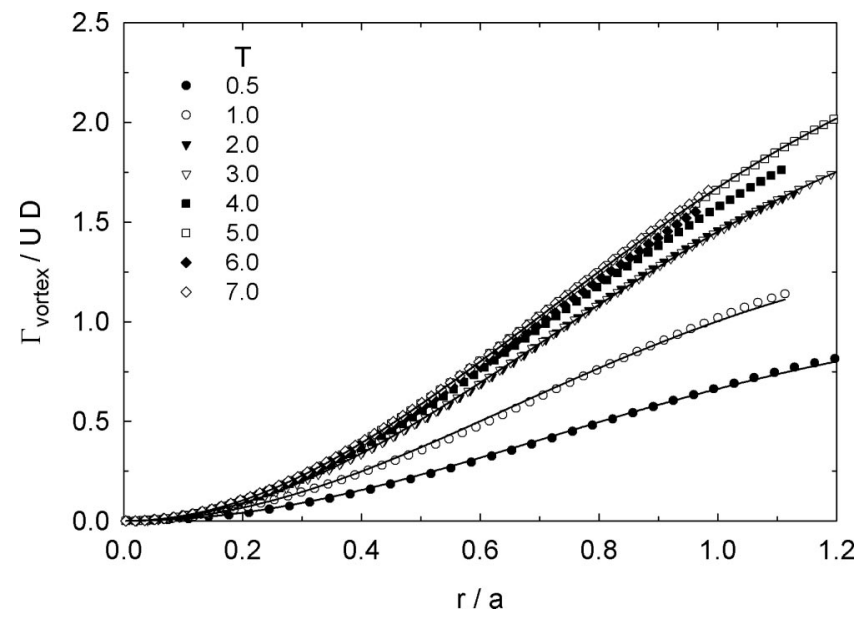

FIG. 15. Vortex ring circulation profiles (symbols) and curve fits (lines) at various times during the flow startup.

(see Figs. 3 and 8), the distribution of vortex ring circulation with the distance from the vortex core can be found. Weigand and Gharib ${ }^{28}$ have shown that vortex rings created by a cylindrical generator have Gaussian vorticity profiles associated with the Lamb-Oseen solution. Based on this result, we have fitted the vortex circulations at each instant with an exponential profile of the form

$$
\Gamma_{\text {vortex }}=\Gamma_{0}\left(1-e^{-(r / a)^{2}}\right) .
$$

The vortex circulation based on the circular contours fitted the above expression very well as long as the contour radius was less than either the axial or radial position of the vortex core. At radii of $r=a$ and $2 a$, the vortex circulation is $63 \%$ and $98 \%$ of $\Gamma_{0}$, respectively. The parameter $\Gamma_{0}$ can be taken as the effective vortex circulation had the vortex ring profile been extended to infinity. Similarly, $a$ can be taken as an effective vortex core radius.

The vortex circulation is plotted against contour radius $(r / a)$ in Fig. 15 for $0.5 \leqslant T \leqslant 7$. The symbols are the computed data and the lines are the curve fits. The curve fits were terminated when the radius became equal to the axial or radial position of the vortex core. It is clear from the data in Fig. 15 that the vortex circulation increases with time at first. However, the circulation distributions tend to collapse onto each other for times greater than $T \approx 4$. The collapse indicates that the starting vortex ring is fully formed by this time, and no further increase in the vortex circulation is expected.

The evolution of the effective vortex circulation $\Gamma_{0}$ and effective core radius $a$ are plotted in Fig. 16. Both $\Gamma_{0}$ and $a$ reach a plateau at $4<T<5$. The average value of vortex circulation is $\Gamma_{0}=2.6 U D$ for $4<T<7$, in excellent agreement with the experimentally observed plateau of $2.5 U D$ in Ref. 6. The experimental data in Ref. 6 also indicate that the circulation reaches its maximum value at a time of $T \approx 4$, in agreement with the computational result. The effective core radius has an average value of $a=0.31 D$ for $4.5<T<7$. If the starting vortex is followed up to the breakup point at $T$ $\approx 12$, the vortex ring circulation (computed on vorticity contours that distinguish the vortex ring from the feeding shear layer) turns out to be equal to $\Gamma_{0}$.
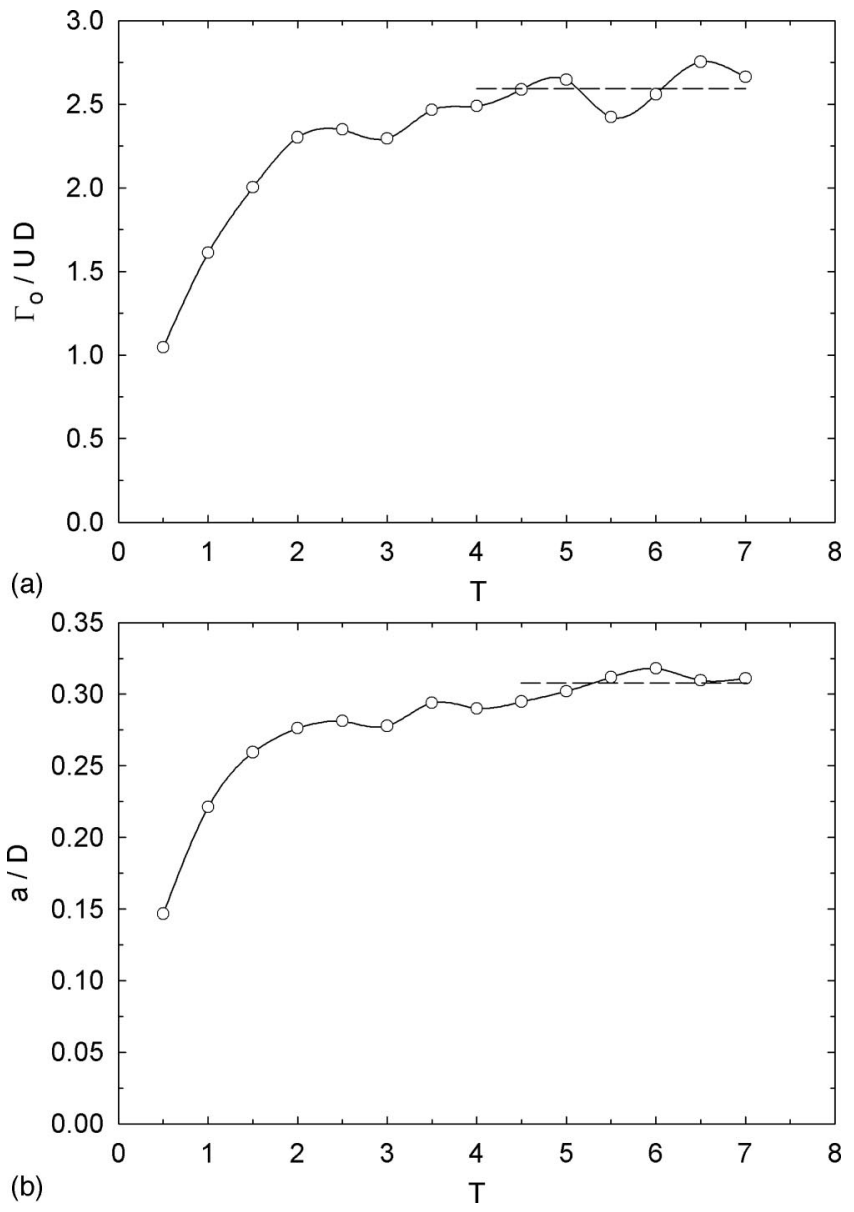

FIG. 16. Evolution of the effective vortex ring circulation $\Gamma_{0}$ in (a), and effective core radius in (b). The dashed lines indicate the average values.

The computed starting vortex ring behind the impulsively started disk also appears to be fully formed at a time of $T \approx 4$, corresponding to a distance of 4 diameters traveled by the disk. This is consistent with the formation of free vortex rings studied by Gharib et al. ${ }^{25}$ A fully formed vortex ring is expected to have a constant celerity, at least for a short period after the formation stage. The axial vortex core position, averaged in the azimuthal direction, is plotted in Fig. 17. The region extending from $T=4$ to 12 fits well to a straight line, revealing a constant celerity for the starting vortex ring. Even after the onset of asymmetry at $T \approx 7$, the mean vortex core position follows the line, indicating that the constant celerity is maintained until the ring breaks up completely. Thus, the starting vortex ring behind an impulsively started disk is fully formed at a time of $T \approx 4$, and it retains its circulation up to a time of $T \approx 12$.

\section{E. Vortex ring breakup}

Perturbations stemming from numerical errors initiate the loss of initial symmetry in the disk wake. No external perturbation was imposed on the flow field since it is difficult to foresee the most natural disturbance to trigger the instability. The instability of the steady flow, not the time evolving flow, past the disk and spheres has been examined in the past. $^{3,29}$ The most unstable mode in the steady base flow has 


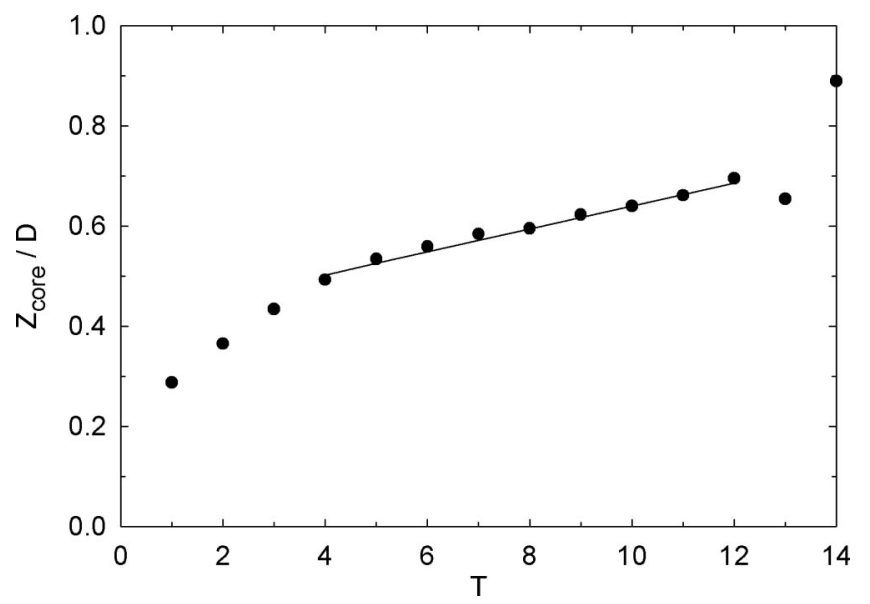

FIG. 17. Azimuthally-averaged vortex core position as a function of time. The straight line indicates a constant vortex ring celerity after $T=4$.

been shown to be helical. Shirayama ${ }^{30}$ has studied the instability of a starting sphere in a numerical study, and has related the topological changes in the vorticity field to the separation pattern on the sphere.

The onset of three-dimensionality in the starting disk case has been examined in Ref. 6 in terms of the Widnall instability, ${ }^{18}$ and the number of the waves on the vortex core is found to be consistent with the theory. As discussed earlier, the computed iso-pressure and iso-vorticity surfaces also indicate that the disk vortex ring experiences the Widnall instability. The number of waves on the computed vortex ring was 3-4, due to the thick vortex cores $(a / R$ $=0.5-0.6$ ). Here, we take advantage of the available pressure field to examine the pressure distribution within the core, the azimuthal flow along the vortex core, and the implications of the azimuthal gradients on the onset of threedimensionality.

Pressure contours in two perpendicular planes are shown in Fig. 18 at several instants during the vortex ring breakup. The minimum contour has a $C_{p}=-2.0$, and the blank regions in the vortex cores have pressures less than this value. The left column shows contours in the $y-z$ plane whereas the $x-z$ plane is shown in the right column. A consequence of the instability waves was the development of azimuthal pressure gradients along the core. This is consistent with Maxworthy's observations. ${ }^{31}$ At $T=9$, the lower core in the $x-z$ plane has a higher pressure than the rest. As time evolves, the instability wave amplitude increased leading to a clear tilting of the vortex ring. Meanwhile, the higher pressure in the lower portion of the $x-z$ plane persisted. The pressure gradient brings about an azimuthal flow which redistributed the initially axisymmteric vorticity. Consequently, the core at $T$ $=13$ was severely distorted in the $y-z$ plane. Finally at $T$ $=15$, multiple minima appeared in the pressure contours and any resemblance of a coherent vortex ring was lost. After the starting ring broke up, smaller scale vortices formed close to the disk. This is indicated by the low-pressure regions, especially visible on the top of the $x-z$ plane.

The presence of the azimuthal flow along the vortex core is revealed by the instantaneous streamlines originating from
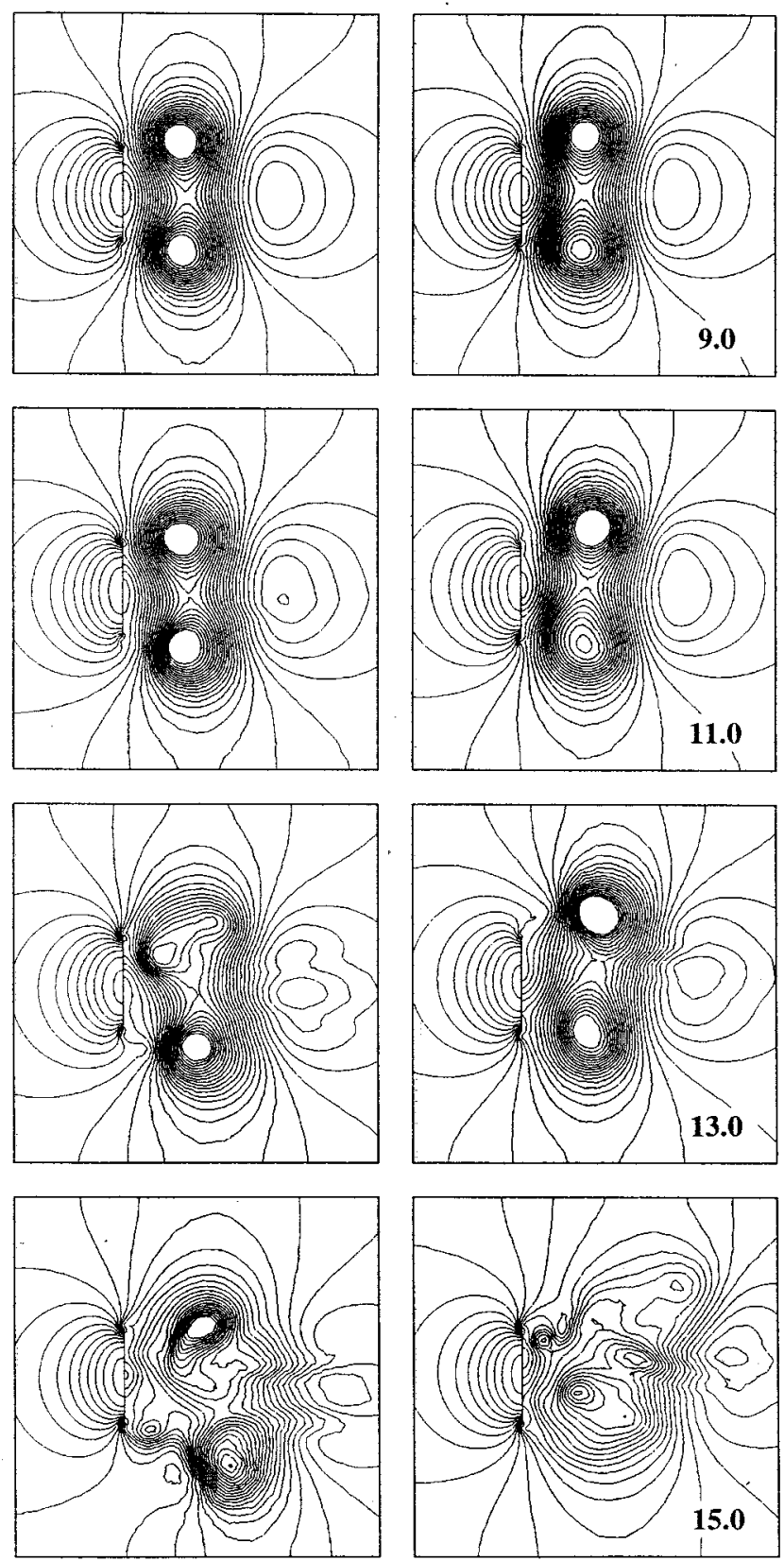

FIG. 18. Development of iso-pressure contours in two perpendicular planes during the ring breakup. The $C_{p}$ contour step is 0.3 . The numbers indicate the nondimensional time $T$.

ten equally spaced points on the $x$-axis along the disk. In the absence of any azimuthal flow, these streamlines would remain primarily in the $x-z$ plane and reveal the recirculation bubble structure (see Fig. 10). On the other hand, a threedimensional structure is expected with azimuthal flow. Instantaneous 3-D streamlines at $T=10.0$ and 12.0 are depicted in Fig. 19 in $y-z$ and $x-z$ views. At $T=10.0$, the threedimensional structure of the streamlines is clearly visible even though the streamlines originated along the $x$-axis. The streamlines have migrated out of the $x-z$ and into the $y-z$ plane, indicating the presence of azimuthal flow. At $T$ $=12.0$, transport along the core and out of the $x-z$ plane becomes even more apparent. The streamlines moved along 

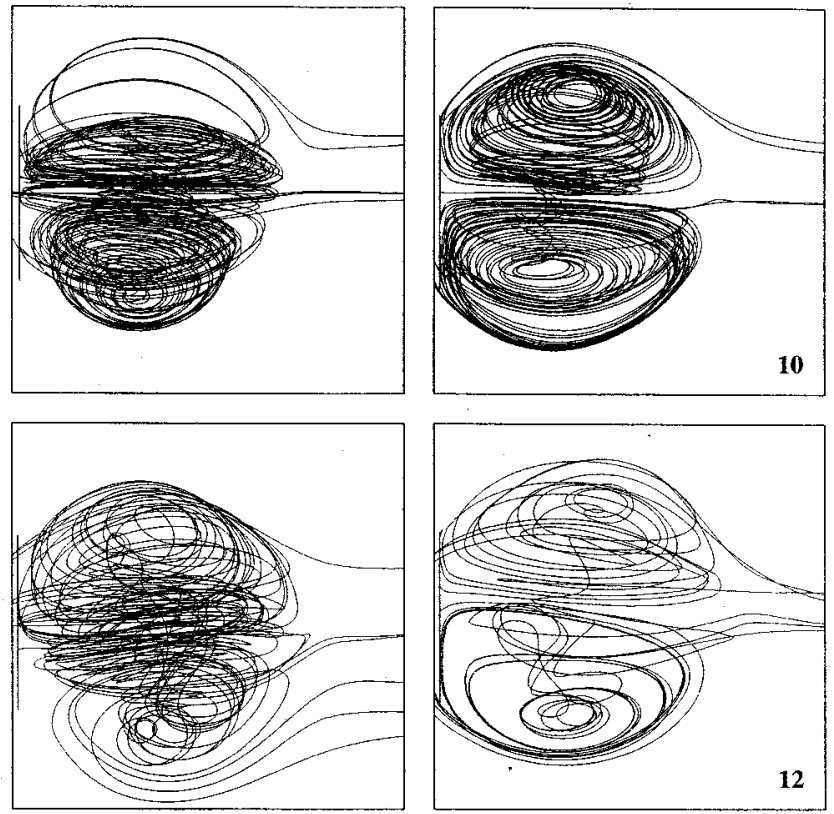

FIG. 19. Streamlines originating from ten equally spaced points on the $x$-axis (on the right) along the disk at $T=10$ and 12 . The $y-z$ view is on the left and the $x-z$ view is on the right.

the vortex core in the pressure gradient direction before leaving the recirculation bubble. Thus, it appears that the azimuthal flow and pressure gradients play a key role in the breakup of the starting vortex ring.

\section{F. Steady-state phase}

The evolution of the flow field following the initial phase was marked by a transition phase before the flow settled into its steady state. During the transition phase, the remnants of the starting vortex ring were shed and smaller three-dimensional vortex filaments formed quite close to the rear of the disk. A cross-sectional view of these vortices is visible in Figs. 8 and 18 at $T=15$. Small-scale vortices are also discernible in the flow visualization experiments of Balligand and Higuchi (see Fig. 60 in Ref. 4) accompanying the breakup and shedding of the starting vortex. Eventually these smaller vortices gave way to a large open wake structure with the separating shear layers forming large-scale vortices. The flow on the upstream side of the disk remained the same while the wake went through the transition phase and finally settled into its steady state.

The drag coefficient history for the entire computational time is shown in Fig. 20. During the transition, the drag coefficient increased to a maximum value at $T=19.2$ before decreasing to a plateau at $1.14 \pm 1 \%$. The drag peak in the transition phase was due to the close proximity of the small vortices to the disk surface. The drag coefficient was nearly constant for times greater than about $T=30$, with minute fluctuations. The steady-state drag was approximately $75 \%$ greater than the minimum drag in the startup phase. Hoerner $^{32}$ has reported a drag coefficient of 1.17 for a solid disk in a steady stream, with the drag being nearly independent of the Reynolds number for values beyond a few thou-

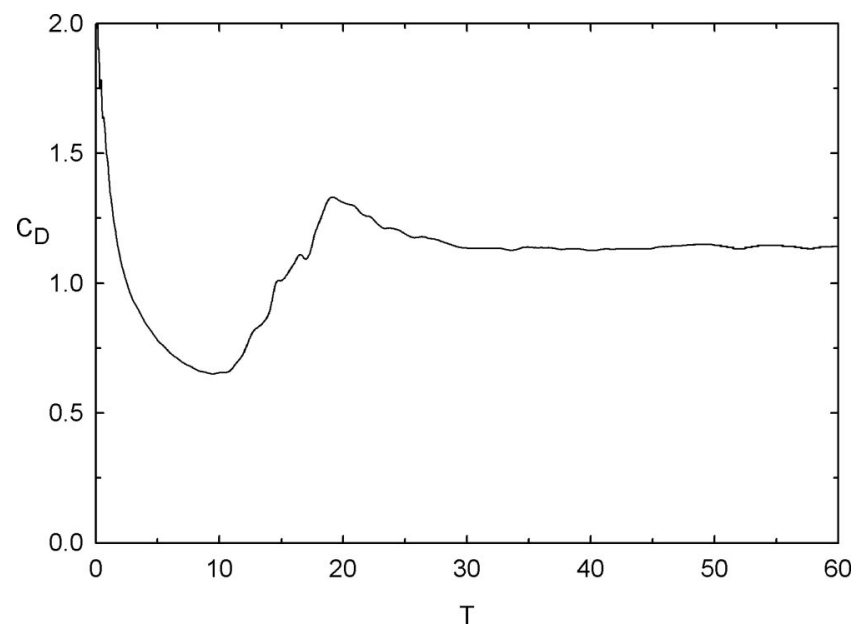

FIG. 20. Drag coefficient history.

sands. The computed average steady-state drag is within 3\% of the reported experimental data, further demonstrating the fidelity of the computational data.

The base pressure coefficient history up to a time of $T$ $=60$ is shown in Fig. 21. The base pressure coefficient increased from a large negative value at the onset of flow to a maximum of +0.35 at $T=9.5$ during the vortex ring instability, before decreasing to a minimum at $T=20$. This minimum base pressure corresponds to the drag peak and is caused by the formation of small vortices close to the disk. After these vortices dissipated, the base pressure increased to its steady-state value of $-0.46 \pm 5 \%$ for times greater than $T=30$. Interestingly, the base pressure coefficient fluctuated about the mean in the steady-state phase. An average value of -0.45 has been reported by Roberts ${ }^{33}$ for the base pressure coefficient of a disk in steady flow at a Reynolds number of $1.3 \times 10^{6}$. The computed average base pressure coefficient in the steady phase matches the experimental data by $2 \%$.

The pressure distribution on the disk surface averaged from $T=30$ to 60 is plotted in Fig. 22. The average pressure distribution as measured by Roberts ${ }^{33}$ at Reynolds number $1.3 \times 10^{6}$ is also included in the plot (symbols). The pressure

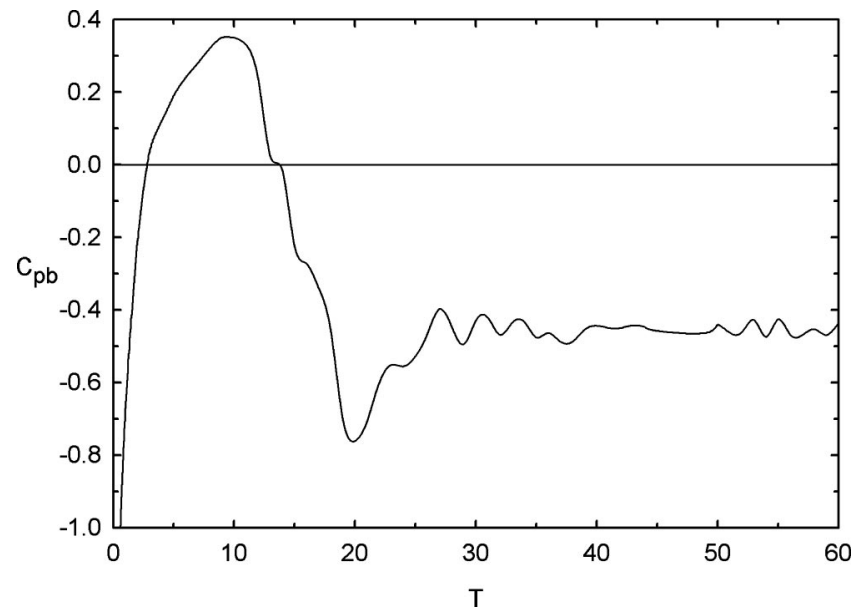

FIG. 21. Base pressure coefficient history. 


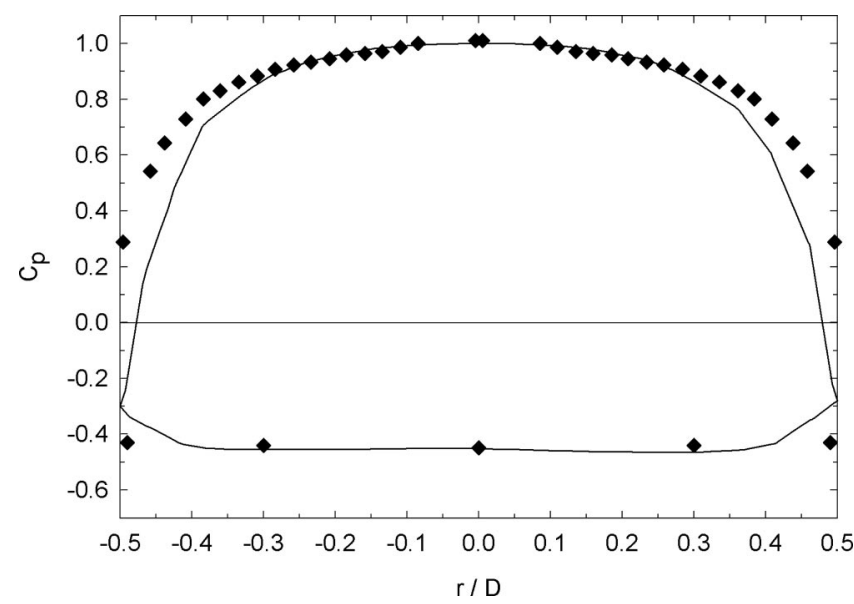

FIG. 22. A comparison of the average pressure coefficient distribution (curve) with the experimental data from Roberts (Ref. 33) (symbols).

coefficient over the majority of the disk backside is uniform, in excellent agreement with the measured pressure distribution. The pressure coefficient over the upstream face is nearly uniform over the central portion of the disk, and decreases at $r / D \sim 0.3$. The data of Roberts decrease closer to the disk lip. The agreement between the computed and experimental pressure distributions is good considering that the experimental data are at a greater Reynolds number.

The nearly constant drag during the steady-state phase of the flow brings up the question of whether vortex shedding was captured in the computations. To answer this question iso-pressure surfaces in the near wake at the time $T=54$ are rendered in Fig. 23. The extent of the images is $2.6 D$. Two views of the elements having pressure coefficients less than -0.65 are shown in this figure. The geometry of these isopressure surfaces seems to suggest a complex vortex patterns at somewhat regular intervals in the wake. Note the streamwise vortex filaments interlaced with the crescent-shaped azimuthal vortices. The absence of shear layer in this figure is due to the visualization scheme that masks the shear layer vorticity near the disk perimeter.

Previous analysis ${ }^{3}$ and experimental work ${ }^{1,2}$ on disks in steady flow at similar Reynolds numbers has shown that the dominant vortex shedding mode is helical. The experimental data were obtained at locations 3 to $9 D$ downstream of the disk. Although the structure of the shed vortices in our computations is different than that in the previous investigations, the two notions of vortex shedding are not necessarily inconsistent. Our crescent-shaped structures exist in the very near field $(z<3 D)$, whereas the previous work has focused on a region farther downstream. It is quite likely that the crescentshaped vortex structures in the very near field will coalesce with the emerging streamwise filaments to form helical structures farther downstream, analogous to that in the model of Howe et al. ${ }^{34}$

A bluff body geometry resembling that of a fully-inflated parachute canopy has also been examined by the finite element code utilized here. ${ }^{35}$ The flow development is qualitatively similar to that described above; however, both the global and local flow parameters are quantitatively different. A

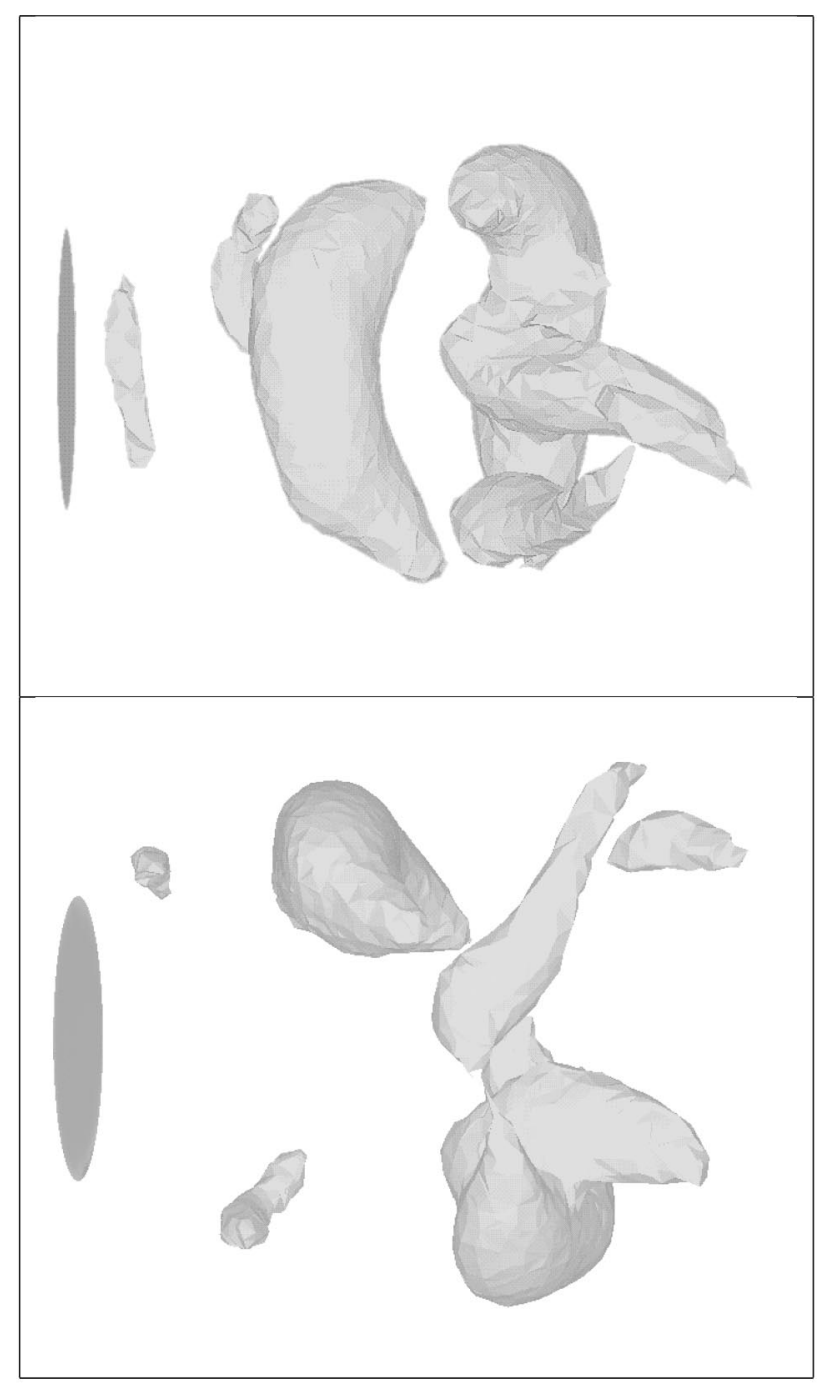

FIG. 23. Two views of the vortex shedding pattern as revealed by isopressure surfaces.

practically important aspect of the flow field around the canopy is the side force induced by the breakup of the starting vortex. The side force and other practically oriented details are emphasized in Ref. 35 whereas the basic flow physics is highlighted in the present article.

\section{REFINED MESH}

To ascertain that the computed data and the resulting flow features are not a function of the mesh refinement, two other finite element meshes were generated. The mesh used for the data reported above, hereafter labeled the "base" mesh, had 708,594 elements with 114,050 nodes. A finer mesh had nearly twice as many elements $(1,304,169$ elements with 209,410 nodes) as the base mesh, and a coarser mesh had about $1 / 7$ th as few elements. The coarser mesh was generated to determine how reduced spatial resolution affects the computed results. It turned out that although the results from the coarser mesh had qualitatively similar behavior as the base mesh, the quantitative values were noticeably different from the base mesh data. For example, the steady-state 


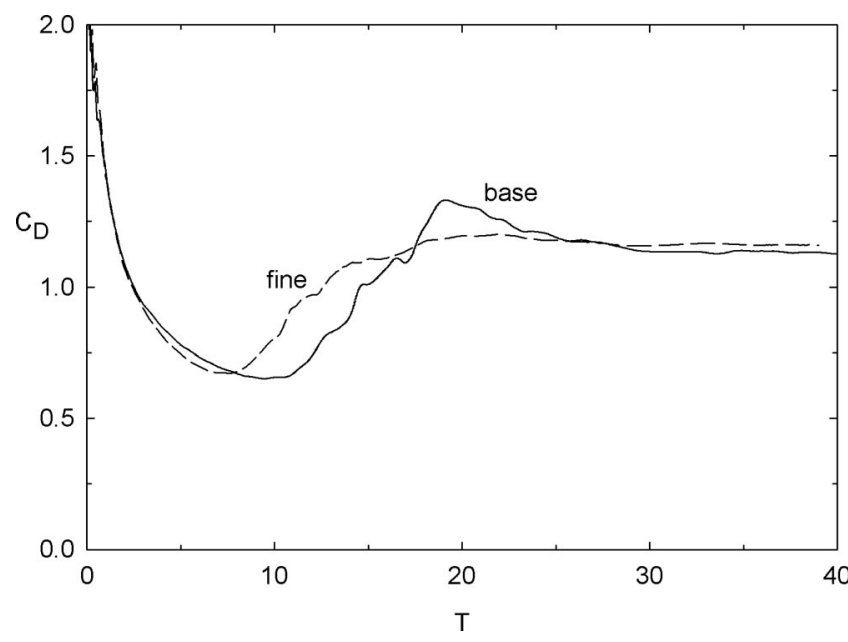

FIG. 24. A comparison of the drag coefficient for the base and fine mesh computations.

drag was smaller and the base pressure coefficient higher than the ones presented above. The coarser mesh data are not discussed further since the spatial resolution was deemed too deficient to accurately simulate the flow field.

The drag time history of the finer mesh and base computations is compared in Fig. 24 for the period $0<T<39$. The two drag coefficients are nearly identical from the start to the time of $5 D / U$, and for times greater than $25 D / U$. The minimum drag computed by the two meshes is within $3 \%$ of each other though the instability and breakup of the starting vortex ring is seen earlier in the data from the fine mesh. During the transition phase, the drag also rises and levels out earlier with the fine mesh as compared to the base mesh. The average drag coefficient in the steady-state phase differs by less than $2 \%$ between the base and fine meshes. Thus, we believe, the drag behavior in the startup and steady-state phases is mesh independent. Although the drag values in the transition phase appear to depend on the mesh refinement, at least for the cases studied here, the overall trends are alike. This is not unexpected since flow transitions are quite sensitive to the resolution, and always require extremely fine resolution to capture accurately.

The base pressure coefficient time history for the base and fine meshes are compared in Fig. 25. As with the drag behavior, the data from both meshes follow similar trends in the startup and steady-state phases. The average $C_{p b}$ in the steady-state phase differed by approximately $2 \%$ between the base and fine meshes. Just as for the drag coefficient, the base pressure coefficients computed by the two meshes were different during the starting vortex ring breakup and the transition phase even though the trends were comparable. The finer mesh also resulted in an initially greater peak $C_{p b}$.

Drag and base pressure coefficients are global measures and not adequate measures of the local flow behavior. Since the starting vortex ring dominates the startup phase, the vortex core position is a good indicator of the details of the local flow field. The temporal evolution of the vortex core position obtained from the minimum pressure location in a crosssectional plane is shown in Fig. 26 for the base and fine mesh. The radial position of the vortex core is quite close for

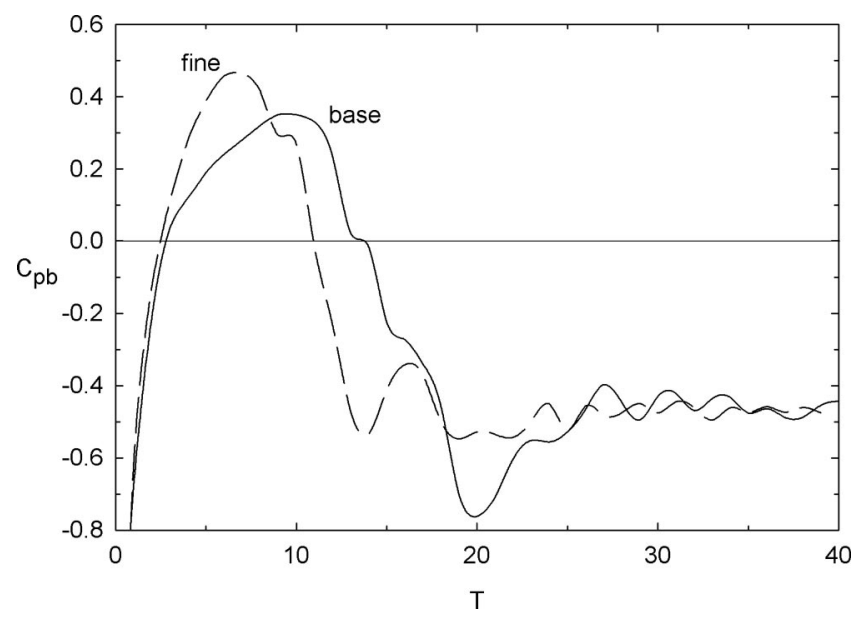

FIG. 25. The base pressure coefficient resulting from the base and fine mesh computations.

the two meshes throughout the startup phase. The axial vortex core position computed by the fine mesh follows closely that of the base mesh up to a time of about $8 D / U$. The onset of instability waves on the starting vortex ring began earlier with the fine mesh. The breakup and shedding of the starting vortex also occurred earlier with the fine mesh at $T \sim 12$ as compared with $T \sim 14$ for the base mesh. The earlier breakup
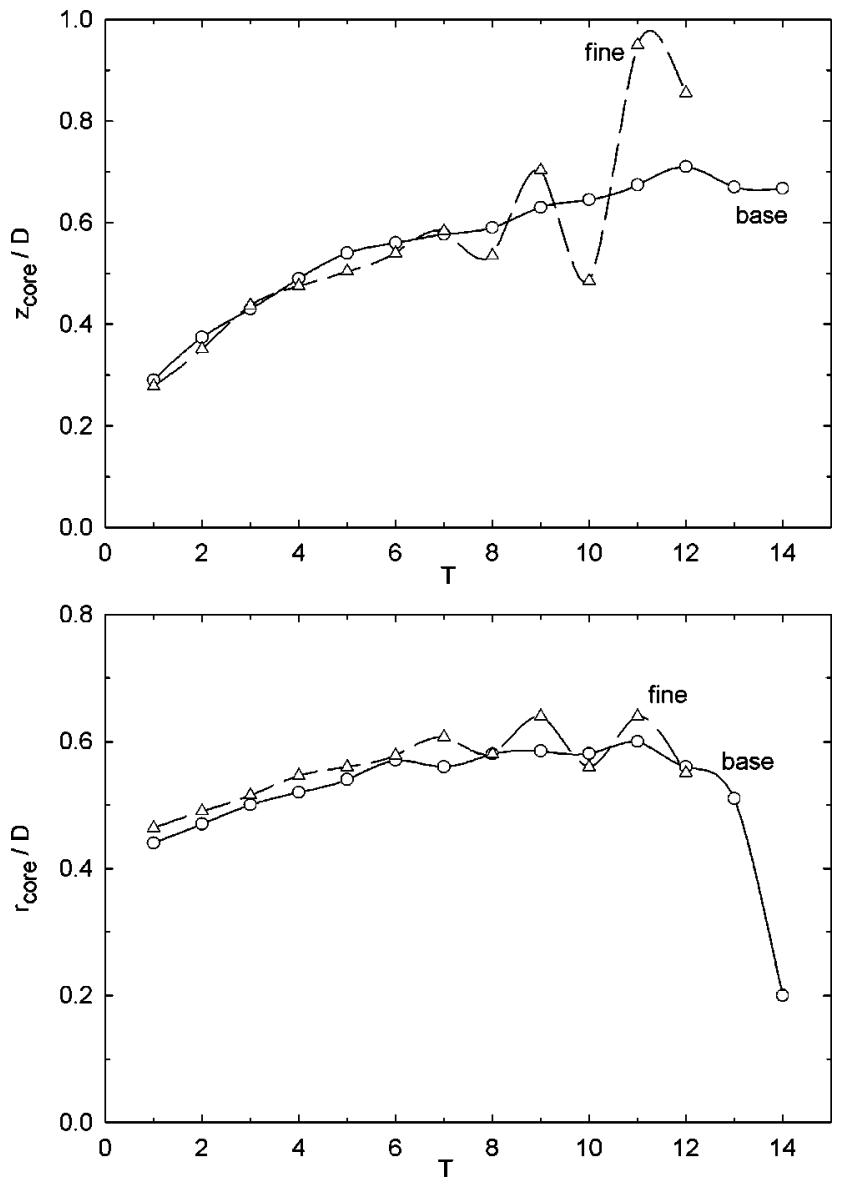

FIG. 26. The axial and radial position of the starting vortex core during the startup phase as computed by the base and fine meshes. 
of the starting vortex ring in the fine mesh case is consistent with the comparisons of drag and base pressure coefficients presented above.

Thus, we suggest that the results computed with the base mesh and presented in Sec. III are mesh independent during the axisymmetric and steady-state phases. The overall trends in the transition phase also appear to be reliable with the caveat that the breakup and shedding of the starting vortex ring and the subsequent establishment of the open wake occurs earlier with the finer mesh. This phase of the flow evolution would require further scrutiny.

\section{CONCLUSIONS}

The ability to accurately simulate the three-dimensional viscous flow field in the near wake of an impulsively started disk of infinitesimal thickness by a finite element code has been demonstrated. The separated shear layer created an axisymmetric starting vortex ring within the wake circulation bubble, and the pressure gradient produced by the starting vortex resulted in the creation of secondary vorticity on the disk backside. As time evolved, the vortex ring was stretched in the axial direction. The peak vorticity in the starting vortex core decreased while the ring was still symmetric. The minimum pressure location in the vortex core was inward of the peak vorticity. During the symmetric phase, the vortex ring reached a constant spreading rate.

The radial circulation profiles of the starting vortex fitted well to a Gaussian expression associated with the LambOseen solution. The circulation profiles collapsed onto each other after a time of $T \approx 4$, consistent with the formation number arguments of free vortex rings by Gharib et al. ${ }^{25}$ The fully formed, thick-cored starting vortex ring had a circulation of $\approx 2.6 U D$, and an effective core radius of $a \approx 0.3 D$. The computed circulation is in good agreement with the experimental data of Balligand. ${ }^{5-7}$ The vortex ring had a constant celerity and the vorticity extended all the way to the symmetry axis after $T \approx 4$. The rate of increase of wake energy and impulse was smaller than that predicted by the slugflow model while the circulation increased more rapidly than the slug-flow model.

During the startup phase, drag decreased and went through a minimum while the base pressure increased and obtained a maximum. The base pressure coefficient became positive and remained so until the breakup and shedding of the starting vortex. The Widnall instability caused the vortex ring to become wavy and eventually led to the breakup of the starting vortex. Complex vortex interactions followed and the remnants of the starting vortex ring were shed in a time of about $14 D / U$. The starting vortex ring breakup was concurrent with the appearance of an azimuthal pressure gradient and flow in the vortex core. Subsequent to the breakup of the starting vortex ring, the flow became three-dimensional and settled into an open wake. The mean drag and base pressure coefficients were nearly constant $( \pm 5 \%)$ after a time of approximately $30 D / U$. The computed drag and base pressure coefficients as well as the pressure distribution on the disk surface matched very well against experimental values.
Vortex shedding in the form of crescent-shaped filaments was observed in the near wake despite the constant drag coefficient in the steady state.

\section{ACKNOWLEDGMENTS}

This work was supported by the Natick Soldier Center (NSC) under the auspices of the U.S. Army Research Office Scientific Services program administered by Battelle (Delivery Order 437, Contract No. DAAH04-96-C-0086), and by ARO Grant No. DAAG55-98-1-0171. The encouragement of Dr. Calvin Lee and Richard Benney during the first author's visit to the Airdrop Technology Team at NSC is appreciated. This work was also supported by Professor T. Tezduyar and his collaborators through their development of the research code that was utilized in the numerical simulation presented here.

${ }^{1}$ H. V. Fuchs, E. Mercker, and U. Michel, "Large-scale coherent structures in the wake of axisymmetric bodies," J. Fluid Mech. 93, 185 (1979).

${ }^{2}$ E. Berger, D. Scholz, and M. Schumm, "Coherent vortex structures in the wake of a sphere and a circular disk at rest and under forced vibrations," J. Fluids Struct. 4, 231 (1990).

${ }^{3}$ R. Natarjan and A. Acrivos, "The instability of the steady flow past spheres and disks," J. Fluid Mech. 254, 323 (1993).

${ }^{4} \mathrm{H}$. Balligand and H. Higuchi, "Experimental investigation of the wake behind a solid disk," Sandia National Laboratories Report SAND 90-7083, Albuquerque, NM, 1993, p. 168.

${ }^{5}$ H. Balligand, J. Zhang, and H. Higuchi, "Wake structure behind a disk started in uniform or near-impulsive accelerations," Proceedings of the ASME Fluids Engineering Division Annual Summer Meeting, Paper FEDSM98-5163, 1998.

${ }^{6}$ H. Balligand, "Unsteady wake structure behind a solid disk," Ph.D. thesis, Syracuse University, June 2000.

${ }^{7}$ H. Higuchi and H. Balligand, "Circulation time history and onset of threedimensional vortex shedding in the wake behind a disk," Proceedings of the 2nd International Symposium on Turbulence and Shear Flow Phenomena, 2001, Vol. 1, pp. 301-306.

${ }^{8}$ Q.-X. Lian and Z. Huang, "Starting flow and structures of the starting vortex behind bluff bodies with sharp edges," Exp. Fluids 8, 95 (1989).

${ }^{9}$ J. H. Strickland, "Prediction method for unsteady axisymmetric flow over parachutes," J. Aircr. 31, 637 (1994).

${ }^{10}$ H. Higuchi, H. Balligand, and J. H. Strickland, "Numerical and experimental investigations of the flow over a disk undergoing unsteady motion," J. Fluids Struct. 10, 705 (1996).

${ }^{11} \mathrm{~S}$. Shirayama and K. Kuwahara, "Computation of flow past a parachute by a three-dimensional vortex method," AIAA Paper 86-0350, January 1986.

${ }^{12}$ T. E. Tezduyar, "Stabilized finite element formulations for incompressible flow computations," Adv. Appl. Mech. 28, 1 (1991).

${ }^{13}$ J. Smagorinsky, "General circulation experiments with the primitive equations," Mon. Weather Rev. 91, 99 (1963).

${ }^{14}$ T. E. Tezduyar and Y. Osawa, "Methods for parallel computation of complex flow problems," Parallel Comput. 25, 2039 (1999).

${ }^{15}$ V. Kalro and T. E. Tezduyar, "Parallel 3D computation of unsteady flows around a circular cylinder," Parallel Comput. 23, 1235 (1997).

${ }^{16}$ Y. Osawa and T. Tezduyar, "A multi-domain method for 3D computation of wake flow behind a circular cylinder," Comput. Fluid Dyn. J. 8, 296 (1999).

${ }^{17}$ A. A. Johnson and T. E. Tezduyar, "Parallel computation of incompressible flows with complex geometries," Int. J. Numer. Methods Fluids 24, 1321 (1997).

${ }^{18}$ S. E. Widnall, "The structure and dynamics of vortex filaments," Annu. Rev. Fluid Mech. 7, 141 (1975).

${ }^{19}$ P. G. Saffman, "The number of waves on unstable vortex rings," J. Fluid Mech. 84, 625 (1978).

${ }^{20}$ K. Chua, D. Lisoski, A. Leonard, and A. Roshko, "A numerical and experimental investigation of separated flow past an oscillating flat plate," ASME International Symposium on Nonsteady Fluid Dynamics, FED-Vol. 92, 1990, pp. 455-464.

${ }^{21}$ P. Koumoutsakos and D. Shiels, "Simulations of the viscous flow normal 
to an impulsively started and uniformly accelerated flat plate," J. Fluid Mech. 328, 177 (1996).

${ }^{22}$ D. Pullin and A. E. Perry, "Some flow visualization experiments on the starting vortex," J. Fluid Mech. 88, 239 (1980).

${ }^{23}$ K. Shariff and A. Leonard, "Vortex rings," Annu. Rev. Fluid Mech. 24, 235 (1992).

${ }^{24} \mathrm{~N}$. Didden, "On the formation of vortex rings: Rolling-up and production of circulation," Z. Angew. Math. Phys. 30, 101 (1979).

${ }^{25}$ M. Gharib, E. Rambod, and K. Shariff, “A universal time scale for vortex ring formation," J. Fluid Mech. 360, 121 (1998).

${ }^{26} \mathrm{~K}$. Mohseni and M. Gharib, "A model for universal time scale of vortex rings," Phys. Fluids 10, 2436 (1998).

${ }^{27}$ K. Mohseni, H. Ran, and T. Colonius, "Numerical experiments on vortex ring formation," J. Fluid Mech. 430, 267 (2001).

${ }^{28}$ A. Weigand and M. Gharib, "On the evolution of laminar vortex rings," Exp. Fluids 22, 447 (1997).
${ }^{29}$ I. Kim and A. J. Pearlstein, "Stability of flow past a sphere," J. Fluid Mech. 211, 73 (1990).

${ }^{30}$ S. Shirayama, "Flow past a sphere: Topological transitions of the vorticity field," AIAA J. 30, 349 (1992).

${ }^{31}$ T. Maxworthy, "Some experimental studies of vortex rings," J. Fluid Mech. 81, 465 (1977).

${ }^{32}$ S. F. Hoerner, Fluid-Dynamic Drag, published by the author, Midland Park, NJ, 1965.

${ }^{33}$ B. W. Roberts, "Drag and pressure distribution on a family of porous, slotted disks," J. Aircr. 17, 393 (1980).

${ }^{34}$ M. S. Howe, G. C. Lauchle, and J. Wang, "Aerodynamic lift and drag fluctuations of a sphere," J. Fluid Mech. 436, 41 (2001).

${ }^{35}$ H. Johari, K. Stein, and T. Tezduyar, "Impulsively started flow about a rigid parachute canopy,' J. Aircr. 38, 1102 (2001). 